\title{
Catálogo de Oligochaeta Microdrili en la Colección de Invertebrados - Fundación Miguel Lillo (Tucumán, Argentina)
}

\author{
Catalog of Oligochaeta Microdrili in the Invertebrates Collection \\ - Miguel Lillo Foundation (Tucumán, Argentina)
}

\section{Fátima Romero}

Instituto de Invertebrados, Fundación Miguel Lillo, Miguel Lillo 251, (4000) San Miguel de Tucumán, Tucumán, Argentina. Correo electrónico: vfromero@lillo.org.ar

\section{RESUMEN}

Los Oligochaeta acuáticos o Microdrili representan un componente importante de las comunidades de agua dulce en todo el mundo, pero han sido ignorados por los ecólogos y hay poca información sobre su distribución así como sus preferencias de hábitats. Son considerados importantes indicadores biológicos de condiciones ambientales y en Sudamérica el conocimiento es escaso y aunque se considera que fueron descriptas unas 171 especies hasta el presente no hay un registro preciso. El objetivo de este trabajo es presentar el catálogo de los Oligochaeta Microdrili de la Colección Helmintológica de la Fundación Miguel Lillo (CH-O-FML) y. rescatar valiosa información inédita realizando una contribución al conocimiento de estos organismos. El material en su gran mayoría proviene de intensas colectas realizadas en las regiones NOA y NEA pero también se encuentran representadas casi todas las provincias de Argentina además de otros países de Sudamérica. La colección estudiada está representado por las familias Naididae y Enchytraeidae, con 1lgéneros y 16 especies. Se provee además información de distribución y hábitat para cada uno de ellos.

Palabras clave - Annelida, lombrices acuáticas, Argentina, lista de géneros.

\footnotetext{
> Ref. bibliográfica: Romero, F. 2019. "Catálogo de Oligochaeta Microdrili en la Colección de Invertebrados - Fundación Miguel Lillo (Tucumán, Argentina)". Acta zoológica lilloana 63 (1): 14-49. Fundación Miguel Lillo, Tucumán, Argentina. D.O.I.: https://doi.org/10.30550/j.azl/2019.63.1/2

> Recibido: 12/04/19 - Aceptado: 13/05/19

> URL de la revista: http://actazoologica.lillo.org.ar OPEN Access Commons Atribución - No Comercial - Sin Obra Derivada 4.0 Internacional.
} 


\begin{abstract}
Aquatic Oligochaeta or Microdrili represent an important component of freshwater communities around the world, but have been ignored by ecologists and there is little information about their distribution as well as their habitat preferences. They are considered important biological indicators of environmental conditions and in South America knowledge is scarce and although it is considered that 171 species were described so far, there is no precise record. The objective of this work is to present the catalog of the Oligochaeta Microdrili of the Helminthological Collection of the Miguel Lillo Foundation (CH-O-FML) and to rescue valuable unpublished information making a contribution to the knowledge of these organisms. The vast majority of the material comes from intensive collections in the NOA and NEA regions, but almost all the provinces of Argentina and other countries in South America are also represented. The collection studied is represented by the families Naididae and Enchytraeidae, with 11 genera and 16 species. Distribution and habitat information is also provided for each one of them.
\end{abstract}

Keywords - Annelida, aquatic worms, Argentina, list of genera.

\title{
INTRODUCCIÓN
}

Existen hasta el presente 5000 especies válidas de Oligochaeta, de las cuales un tercio aproximadamente son acuáticas o semiacuáticas con 1100 especies de agua dulce y 600 especies marinas o estuarinas (Erséus, 2005; Martin, Martinez-Ansemil, Pinder, Timm, Wetzel, 2008; 2016). Basándose en su tamaño Benham (1890) los divide en Megadrilos ( $>25 \mathrm{~mm}$ de longitud) y Microdrilos ( $<25 \mathrm{~mm}$ ); estos últimos también denominados limnícolas, están representados por 13 familias (incluyendo a los Enchytraeidae que incluyen mayor número de especies terrestres) y viven en agua dulce (ríos y arroyos) además de hábitats salobres y marinos.

Los Oligochaeta acuáticos o Microdrili representan un componente importante de las comunidades de agua dulce en todo el mundo, pero han sido ignorados por los ecólogos y hay poca información sobre su distribución así como sus preferencias de hábitats. Presentan una alta diversidad de géneros y especies y son muy abundantes en distintos tipos de ambientes formando parte de distintas comunidades, tales como bentos, pleuston y perifiton. Biológicamente, reflejan claramente los cambios producidos dentro de un ecosistema reduciendo o ampliando sus ciclos biológicos y estrategias de reproducción o presentando cambios en la abundancia y dinámica de las diferentes poblaciones que caracterizan un ambiente. Algunos géneros presentan altos grados de adaptación que les permiten responder de forma plástica a cuestiones ambientales y evolutivas (Rodríguez y Reynoldson, 2011). Es por ello que son considerados por numerosos autores a nivel mundial (Hellawell, 1986; Rosenberg y Resh, 1993; Traunspurger y Drews, 1996) como indicadores biológicos de importante valor diagnóstico de las condiciones ambientales. Esto se debe principalmente a que poseen una alta tolerancia a un amplio rango de variaciones ambientales y princi- 
palmente a su ciclo de vida que se completa totalmente en el ambiente acuático de este modo solo están expuestos a contaminantes de este medio, proporcionando una buena especificidad del sitio (Rodríguez y Reynoldson, 2011). También constituyen un importante eslabón de la cadena trófica bentónica, dentro de la cual cumplen el rol de: consumidores primarios, descomponedores y modificadores del sustrato además de constituirse como alimento principal para los depredadores (Schwank, 1982). Se encuentran en cualquier tipo de ambientes desde prístinos hasta en aguas eutroficadas y/o contaminadas, donde alcanzan densidades muy elevadas y sirven de alimento para peces bentónicos, renacuajos (anfibios) y aves acuáticas (Aarefjord, Borgstrom, Lien, Milbrink, 1973; Riera, Juget, Martinet., 1991; Gophen, Yehuda, Malinkov, Degani, 1998; Rahman, Verdegem, Nagelkerke, Wahab, Milstein, Verreth, 2006), turbelarios, sanguijuelas, nematodes, cangrejos y larvas de insectos (Loden, 1974; Schürch y Walter, 1978; Brinkhurst, 1980; Kaster, 1989) ya que el $90 \%$ de su peso seco consiste en proteínas y grasas (Yan y Liang, 2004).

A nivel mundial se han descripto aproximadamente unas 1700 especies de Oligochaeta acuáticos pero para Sudamérica el conocimiento es escaso y no hay un registro preciso aunque se considera que fueron descriptas unas 171 especies hasta el presente (Marchese, 2009; Christoffersen, 2010) siendo Argentina y Brasil los países con más estudios (Marchese, 2009; Christoffersen, 2007).

En la Colección Helmintológica de la Fundación Miguel Lillo (CH-O-FML), encontramos ejemplares depositados a partir del año 1897. El material en su gran mayoría está determinado y proviene de intensas colectas realizadas en las regiones NOA y NEA por el Dr. K. Gavrilov eminente especialista en el tema. Pero también se encuentran representadas casi todas las provincias de Argentina además de otros países de Sudamérica (Teisaire, López Aragón, García Moreno, 2005)

El objetivo de este trabajo es rescatar esta valiosa información inédita y realizar una contribución al conocimiento de los Oligochaeta Microdrili depositados en la Colección de la Fundación Miguel Lillo. De este modo y para cumplimentar la recomendación 72 g (4) del código de nomenclatura Zoológica se procedió a realizar el listado de los ejemplares depositados en la misma considerándose también otros datos como los relacionados con las distribuciones que son de gran utilidad para estudios posteriores. Así, el conocimiento de los ejemplares depositados en esta colección proveerá información sobre la distribución, riqueza y otros aspectos ecológicos que se transformarán en herramientas fundamentales para programas de conservación y manejo de áreas naturales.

\section{MATERIALES Y MÉTODOS}

El material estudiado pertenece a la Colección Helmintológica de Oligochaeta de la Fundación Miguel Lillo (CH-O-FML). La colección cuenta con ejemplares fijados con diferentes técnicas (zenker, formol-fleming, bouin, formol y alcohol) y luego conservados en alcohol o formol, además de numerosas preparaciones histológicas. La mayoría del material está en buen estado de conservación. Este catálogo incluye el material que está identificado por el Dr. Konstantin Gavrilov un eminente 
especialista en el tema e incorporaciones recientes de material identificadas por E. Rivas de Pantorrilla y la autora. La colección no cuenta con tipos primarios y solo recientemente (Rodríguez y Fend, 2018) se nombró el Lectotipo de una especie que fue descripta por este especialista (Gavrilov 1955a, 1955b). Para cada ejemplar se brinda una lista detallada con la familia, el nombre de la especie, autor, año de publicación; procedencia (país/estado, provincia, localidad) fecha de colecta, colector y código de la colección. Además se brinda información acerca de su distribución, hábitat y otros aspectos. Las distribuciones actualizadas de cada género y especie se tomaron de los catálogos de Christoffersen (2007, 2009), debido a que todavía no es clara la clasificación dentro de los anélidos, en el presente trabajo se seguirá la clasificación de Dyne y Jamieson (2004) donde Euclitellata es subfilum y Oligochaeta es clase. Para los nuevas provincias de Argentina en las que se registra un género/o especie se utilizó el símbolo * y para las nuevas localidades en cada provincia ${ }^{\star \star}$.

\title{
RESULTADOS
}

Los Oligochaeta Microdrili están representados en la colección Hemintológica de la Fundación Miguel Lillo por las familias Naididae y Enchytraeidae, con 11 géneros y 16 especies colectadas por investigadores de esta institución y ejemplares incorporados a partir de donaciones. No se registran tipos, solo un Lectotipo Paranadrilus descolei. Los géneros y el número de especies presentes en la colección son: Aulophorus (1); Bothrioneurum (2); Chaetogaster (1); Dero (1); Limnodrilus (3); Nais (1); Paranadrilus (1); Pristina (2); Slavina (1), Tubifex (2); Enchytraeus (1).

\section{PHYLUM ANNELIDA \\ SUBPHYLUM EUCLITELLATA \\ CLASE OLIGOCHAETA}

\author{
Orden Haplotaxida \\ Familia Naididae Ehrenberg, 1828
}

Esta familia que fue recientemente redefinida e incluyó a Tubificidae presenta alrededor de 1000 especies descriptas dentro de 7 subfamilias de amplia distribución en todo el mundo (Phallodrilinae, Limnodriloidinae,Telmatodrilinae, Tubificinae, Naidinae, Rhyacodrilinae, y Pristininae) pero solamente las 4 últimas están representadas en Argentina (Erséus, Wetzel, Gustavsson, 2008; Martin, Martinez-Ansemil, Sambugar, 2010). Si bien la familia es cosmopolita, según Christoffersen (2010) existen 152 especies de Naididae registrados para Sudamérica con varios géneros que presentan una distribución tropical o están limitados al Hemisferio Sur. Difieren de los otros Oligoquetos en cuanto a su modo de vida ya que algunos géneros pueden nadar y por lo tanto desplazarse cerca del sedimento de fondo o entre raíces de plantas acuáticas adaptándose a este estilo de vida por el desarrollo de ojos y largas quetas que les permiten nadar. También los encontramos en las proximidades 
de la vegetación acuática (sobre y entre ella) mientras que algunas especies viven enterradas en el sedimento de sustratos lodosos o arenosos (Gavrilov, 1979). Existen especies comensales, parásitas y depredadoras. También pueden encontrarse en aguas epifíticas de bromeliáceas, entre hojas caídas húmedas y en el suelo húmedo y pantanoso. Por otro lado, y con respecto a su posición en la comunidad, es interesante destacar que son frecuentes en ambientes enriquecidos orgánicamente y representan una importante vía en la transferencia de energía pero también de tóxicos de los niveles tróficos inferiores a los superiores. Debido a su sensibilidad a diversos agentes contaminantes, son indicadores potencialmente útiles en el diagnóstico de la calidad ecológica de los sistemas acuáticos (Learner, 1979b; Lang, 1990; Milbrink, 1994).

\section{Género Aulophorus Schmarda, 1861 Aulophorus furcatus Müller, 1774}

Distribución y hábitat.- Principalmente tropical y más abundantes y comunes en el Hemisferio Sur. Esta especie presenta amplia distribución en Centroamérica, América del Norte, Europa, África y Asia a nivel mundial. En Sudamérica la encontramos en Bolivia, Venezuela, Guyana, Surinam, Brasil, Perú, Paraguay. En Argentina en las siguientes provincias: Misiones, Chaco, Corrientes, San Juan, Buenos Aires, Santa Fe, Entre Ríos encontrándose principalmente en lagos, embalses y ríos En el presente trabajo se amplía su distribución a las provincias de Tucumán, Jujuy, Formosa y nuevas localidades en la provincia de Corrientes. Esta especie generalmente vive en lagunas y charcas con una densa vegetación flotante (Marcus, 1944) y también en remansos de ríos. Generalmente se la encuentra asociada a otros organismos como Nymphaea (Marcus, 1944), gasterópodos (Gorni y Alves, 2006), macrófitas y hojas en descomposición (Martins, Martinez-Ansemil, Pinder, Timm, Wetzel, 2008).

Material de colección.—`TUCUMÁN: Jardín del Instituto Miguel Lillo, parte lindante con la calle Miguel Lillo, acuario natural (agua más depósito de hojas) en la base de las hojas de Bromeliaceae planta $N^{\circ}$ 1, San Miguel de Tucumán, 27/XI/1968, varios ejemplares, col: J. C. Viera y K. Gavrilov (CH-O-FML 5552); Jardín del Instituto Miguel Lillo, acuario natural entre las hojas de Bromeliaceae Tillandsia maxima Lillo, San Miguel de Tucumán, 13/III/1969, muchos ejemplares, col: J. C. Viera y K. Gavrilov (CH-O-FML 5754) (CH-O-FML 5755) (CH-O-FML 5756) (CH-O-FML 5757) (CH-O-FML 5758); Jardín del Instituto Miguel Lillo, acuario natural entre las hojas de Bromeliaceae Tillandsia maxima Lillo, en la base de las hojas, San Miguel de Tucumán, 28/III/1969, varios ejemplares, col: J. C. Viera y K. Gavrilov (CH-O-FML 5759); Jardín del Instituto Miguel Lillo, acuario natural entre las hojas de Bromeliaceae Tillandsia maxima Lillo, parte superior, San Miguel de Tucumán, 07/IV/1969, muchos ejemplares, col: J. C. Viera y K. Gavrilov (CH-O-FML 5760); Jardín del Instituto Miguel Lillo, acuario natural entre las hojas de Bromeliaceae Tillandsia maxima Lillo en la base de las hojas, San Miguel de Tucumán, 10/IV/1969,varios ejemplares, col: J. C. Viera y K. Gavrilov (CH-O-FML 5763); Jardín del Instituto Lillo, agua 
de bromeliáceas, San Miguel de Tucumán, 180 ejemplares inmaduros, 14/IX/1979, col: E. Rivas de Pantorrilla (CH-O-FML 7339); Jardín del Instituto Lillo, agua de bromeliáceas junto a la tumba del Dr. M. Lillo, San Miguel de Tucumán, 14/IX/1979, 6 ejemplares, col: E. Rivas de Pantorrilla (CH-O-FML 7343); agua de bromeliáceas en Horco Molle, Yerba buena, 24/IX/1979, 3 ejemplares en cultivo, col: N. Lezcano (CH-O-FML 7344); agua de bromeliáceas en el Funicular de Horco Molle, Yerba buena, 24/IX/1979, 8 ejemplares, col: E. Rivas y N. Lezcano (CH-O-FML 7345), 5 ejemplares- cultivo común (CH-O-FML 7346); Jardín del Instituto Lillo, agua de bromeliáceas junto a la tumba del Dr. M. Lillo, San Miguel de Tucumán, 05/XI/1982, 4 ejemplares jóvenes, col: E. Rivas de Pantorrilla (CH-O-FML 7348), 10 ejemplares adultos y 13 jóvenes (CH-O-FML 7351), 90 ejemplares (CH-O-FML 7355), 3 ejemplares inmaduros (CH-O-FML 7357), 80 ejemplares inmaduros (CH-O-FML 7364), 4 ejemplares sin palpos y con branquias (CH-O-FML 7365), 16 individuos adultos + 37 jóvenes inmaduros (CH-O-FML 7368); Jardín del Instituto Lillo, agua de bromeliáceas, cerca de administración, San Miguel de Tucumán, 06/X/1979, en cultivo, col: N. Lezcano (CH-O-FML 7359); Jardín del Instituto Lillo, agua de bromeliáceas cerca de administración, San Miguel de Tucumán, 18/IX/1979, cultivo, col: E. Rivas (CH-O-FML 7366); río Gastonilla, Iltico, Chicligasta, 07/XII/1982, muchos ejemplares, col: E. Rivas de Pantorrilla (CH-O-FML 7352); río Gastona, ribera del río en bromeliáceas, Chicligasta, 31/XII/1983, muchos ejemplares, col: E. Rivas de Pantorrilla (CH-O-FML 7356); agua de bromeliaceas cerca del río Gastona, Iltico, Chicligasta, 07/XII/1982, ejemplares sin color, col: E. Rivas de Pantorrilla (CH-O-FML 7363).

^JUJUY: bañado a $1 \mathrm{~km}$ después del Arroyo Yuto, ruta entre Caimancito y Yuto, 26/VIII/1968, 93 ejemplares, col: J. C. Viera, V. P. Ruiz y P. Autino (CH-O-FML 5717).

\section{Aulophorus sp.}

Material de colección.- TUCUMÁN: pantano en el camino entre Villa Alberdi y Aguilares , 24/II/1948, 87 ejemplares, col: K. Gavrilov (CH-O-FML 503); 2 ejemplares, col: K. Gavrilov (CH-O-FML 503a); charco en el Jardín del Instituto Lillo, San Miguel de Tucumán, 21/X/1948, varios ejemplares, col: K. Gavrilov (CH-O-FML 774); pantano en el km 52 del camino entre Acheral y Monteros, 07/XII/1948, 3 ejemplares, col: K. Gavrilov y G. Paz (CH-O-FML 877); estanque en Villa Carmela, Tafí Viejo, 18/XI/1979, 122 ejemplares, col: E. Rivas de Pantorrilla (CH-O-FML 7338).

SANTA FE: río Salado en Santo Tomé (por el camino de Santa Fé a Rosario), 23/V/1951, 1 ejemplar, col: K. Gavrilov (CH-O-FML 2276).

^FORMOSA: estero al costado de la ruta antes del puente internacional, Clorinda, 31/X/1973, muchos ejemplares, col: V. P. Ruiz (CH-O-FML 6427) (CH-O-FML 6428) (CH-O-FML 6429).

$\star \star$ CORRIENTES: charco pantanoso en el camino entre Corrientes e Ita ibaté, 29/XII/1949, pocos ejemplares, col: K. Gavrilov y Z. Tomsic (CH-O-FML 1358); 
charco con vegetación en el camino de Corrientes a Posadas, 19/XII/1949, 2 ejemplares, col: K. Gavrilov; Z. Tomsic; T. Apostol; W. T. Angelo (CH-O-FML 1490).

\section{Género Bothrioneurum Stolc, 1886 \\ Bothrioneurum americanum Beddard, 1894}

Distribución y hábitat.- Esta especie presenta distribución netamente sudamericana hasta el presente, encontrándose en Paraguay, Perú, Colombia, Venezuela, Guyana, Brasil y en Argentina en las siguientes provincias: Buenos Aires, Entre Ríos, Santa Fe, Misiones y Santiago del Estero. Se encuentra preferentemente en hábitats lóticos de baja velocidad de corriente en fondos limo-arenosos con bajo contenido de materia orgánica (Zilli, Montalto, Marchese, 2008). En el presente trabajo se amplía su distribución a las provincias de Tucumán y Jujuy.

Material de colección.- ${ }^{\star}$ TUCUMÁN: margen del río Calera, Burruyacu, 01/X/1968, 2 ejemplares maduros, col: T. Fasola (CH-O-FML 5518) (CH-O-FML 5519), 29 ejemplares (CH-O-FML 5520), 1 ejemplar pequeño (CH-O-FML 5521), 7 ejemplares (CH-O-FML 5522), 13 ejemplares (CH-O-FML 5523), 3 ejemplares jóvenes (CH-O-FML 5524), trozos (CH-O-FML 5525); acequia en la estancia del Dr. Vera, $16 \mathrm{~km}$ al este de la ciudad de Trancas, 20/X/1968, 8 ejemplares, col: T. Fasola (CH-O-FML 5531), 8 ejemplares (CH-O-FML 5532), muchos ejemplares (CH-O-FML 5533).

*JUJUY: acequia $3 \mathrm{~km}$ después de la población El Milagro (entre San Pedro de Jujuy y Güemes), 28/VIII/1968, 2 ejemplares, col: J. C. Viera, V. P. Ruiz y P. Autino (CH-O-FML 5707), restos (CH-O-FML 5708a).

Bothrioneurum vejdovskyanum Stolc, 1886.

Distribución y hábitat.- Especie de amplia distribución, se encuentra en Europa, África, Asia y América del Norte. En Sudamérica en Brasil y en Argentina en la provincia de Tucumán. En el presente trabajo se amplía su distribución a las provincias de Salta y Jujuy y a nuevas localidades en la provincia de Tucumán. Habita en ríos, preferentemente en los tramos rectos de la corriente con fondos arenosos o de grava (Schenková, Helešic, Jarkovský, 2006), es tolerante a los procesos de eutrofización encontrándose en sedimentos de sitios degradados (Timm, 2009; Odabaşi, Arslan, Cirik, 2017) y es considerada una especie beta-mesosapróbica (Uzunov, Košel, Sládeèek, 1988).

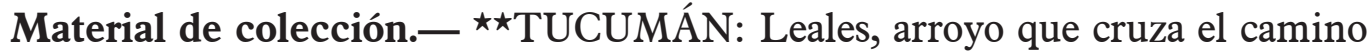
entre Bella Vista y García Fernández, 14/VII/1947, 5 ejemplares, col: K. Gavrilov (CH-O-FML 177); Trancas, Arroyo Tapia, 10/I/1948, 1 ejemplar, col: K. Gavrilov (CH-O-FML 312); San Miguel de Tucumán, acequia en la calle, 13/I/1948, 1 ejemplar, col: R. Golbach (CH-O-FML 318); acequia que cruza el camino entre Bella 
Vista y García Fernández, 26/VI/1948, muchos ejemplares, col: K. Gavrilov (CH-OFML 628); muchos ejemplares (CH-O-FML 628a); muchos ejemplares, con principio de testículos (CH-O-FML 628b); muchos ejemplares, con parásitos (CH-O-FML 628c); varios ejemplares (CH-O-FML 628d); 1 ejemplar anormal con bifurcación (CH-O-FML 628e); 1 ejemplar (CH-O-FML 628f); varios ejemplares (CH-O-FML 628g); Aguada de la Sepultura, camino de Las Arcas a la estancia de Rearte, Trancas, 26/IV/1968, 4 ejemplares, col: T. Fasola (CH-O-FML 5482); algunos ejemplares (CH-O-FML 5483).

^SALTA: arroyo en Termas de Rosario de la Frontera, 18/I/1948, varios ejemplares, col: K. Gavrilov (CH-O-FML 355) (CH-O-FML 355a) (CH-O-FML 355b); 3 ejemplares (CH-O-FML 356); arroyo entre La Silleta y La Merced de Arriba, Rosario de Lerma, 20/I/1948, varios ejemplares, col: K. Gavrilov (CH-O-FML 361); arroyo en el camino entre Salta y San Antonio de los Cobres, aprox. $140 \mathrm{~km}$ de Salta, 28/VII/1948, varios ejemplares, col: K. Gavrilov (CH-O-FML 679).

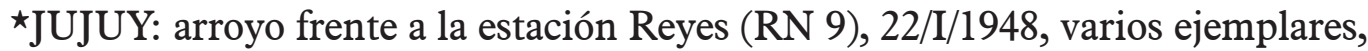
col: K. Gavrilov (CH-O-FML 379) (CH-O-FML 379a); río Yala, Yala, 23/I/1948, pocos ejemplares, col: K. Gavrilov (CH-O-FML 395); lodo de una zanjita de desagüe de un grifo, entre las plantas, Barrio Ortiz, R9, San Salvador de Jujuy, 25/VIII/1967, varios ejemplares, col: K. Gavrilov, J. L. Viera, V. T. Ruiz y T. Fasola (CH-O-FML 5279) (CH-O-FML 5285); margen de una zanjita de desagüe de un grifo, entre las plantas, Barrio Ortiz, R9, San Salvador de Jujuy, 25/VIII/1967, varios ejemplares, col: K. Gavrilov, J. L. Viera, V. T. Ruiz y T. Fasola (CH-O-FML 5294); arroyito a lo largo del camino desde S.S. de Jujuy a Termas de Reyes, $4 \mathrm{~km}$ desde el motel Huaico, 26/VIII/1967, 1 ejemplar, col: K. Gavrilov, J. C. Viera, V. P. Ruiz y T. Fasola (CHO-FML 5353); varios ejemplares (CH-O-FML 5354).

\section{Bothrioneurum sp.}

Distribución y hábitat.- Este género se distribuye principalmente en las regiones tropicales de Sudamérica, habitando arroyos de altura, lodo de pantanos y acequias. Se amplía la distribución del género en Argentina a las provincias de Catamarca, Corrientes, Chaco, Formosa y Mendoza, se registran nuevas localidades para Tucumán, Jujuy, Salta, Santa Fé, Buenos Aires y Entre Ríos

Material de colección.— ${ }^{\star}{ }^{\star}$ TUCUMÁN: arroyo que cruza el camino en el Infiernillo (3020 msnm), Tafí del Valle, 28/I/1948, 7 ejemplares, con espermatóforos, col: K. Gavrilov (CH-O-FML 427); pantano entre Acheral y Santa Lucía, 28/I/1948, 7 ejemplares, col: K. Gavrilov (CH-O-FML 434) (CH-O-FML 434a); pantano sobre el camino entre Acheral y Monteros, 04/III/1949, varios ejemplares, col: K. Gavrilov (CH-O-FML 1877); lodo de un pantano en río Colorado, 23/X/1953, varios ejemplares, col: R. Golbach (CH-O-FML 2377); pantano sobre el camino entre Acheral y Monteros, 12/II/1949, 1 ejemplar, col: K. Gavrilov (CH-O-FML 2382); en el lodo de un arroyo en el río Balderrama, Simoca, 08/III/1956, 3 ejemplares maduros con espermatóforo, col: K. Gavrilov y V. P. Ruiz (CH-O-FML 2846); Arroyo del Toro 
entre García Fernández y Bella Vista, 18/IV/1952, varios ejemplares, col: K. Gavrilov (CH-O-FML 2903); acequia en la estancia del Dr. Vera, $16 \mathrm{~km}$ al este de la ciudad de Trancas, 20/X/1968, 8 ejemplares, col: T. Fasola (CH-O-FML 5534) (CH-O-FML 5535).

$\star \star$ SALTA: arroyo en Termas de Rosario de la Frontera, 13/XII/1947, varios ejemplares, col: A. Willink (CH-O-FML 289); acequia al lado del camino a San José de Cachi (aproximadamente $1 \mathrm{~km}$ en dirección a Cachi), 23/VII/1948, muchos ejemplares, col: K. Gavrilov (CH-O-FML 711) (CH-O-FML 711a) (CH-O-FML 711b); acequia al lado del camino entre Seclantás y Cachi, 23/VII/1948, muchos ejemplares, col: K. Gavrilov (CH-O-FML 713) (CH-O-FML 713a) (CH-O-FML 713b); acequia en Vaqueros (camino de cornisa), 26/VII/1948, varios ejemplares, col: K. Gavrilov (en el limo traído por O. Budin y A. Say) (CH-O-FML 719); acequia que cruza el camino entre Salta y San Antonio de los Cobres, 28/VII/1948, varios ejemplares, col: K. Gavrilov (CH-O-FML 755); arroyo en Escalchi por el camino entre Molinos y Cachi, 23/VII/1948, varios ejemplares, col: K. Gavrilov (CH-O-FML 759); arroyito en Termas de Rosario de la Frontera, 13/XII/1947, col: K.Gavrilov (en limo traído por A. Willink) (CH-O-FML 871); acequia al costado del camino $3 \mathrm{~km}$ después de Güemes hacia Tucumán, 28/VIII/1968, algunos ejemplares, col: J. C.Viera, V. P. Ruiz y P. Autino (CH-O-FML 5584); en el margen del río Bermejo 40 $\mathrm{m}$ antes de los bananales, General José de San Martín, 22/VI/1971, varios ejemplares, col: V. P.Ruiz (CH-O-FML 6036) (CH-O-FML 6037) (CH-O-FML 6038); desagüe al costado de la ruta, Agua Blanca, Oran, 22/VI/1971, algunos ejemplares, col: V. P. Ruiz (CH-O-FML 6058); 2 ejemplares en maduración (CH-O-FML 6059); varios ejemplares (CH-O-FML 6065).

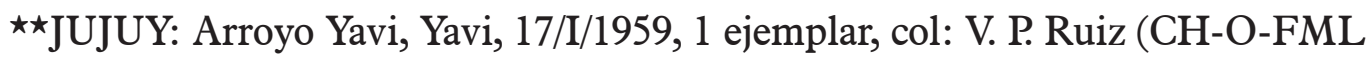
2675), varios ejemplares (CH-O-FML 2676) (CH-O-FML 2677); acequia cruzando el camino en San Juancito (4 km antes después del Cuarteadero), El Carmen, 28/VIII/1968, 1 ejemplar maduro, col: J. C. Viera, V. P. Ruiz y P. Autino (CH-OFML 5601), 5 ejemplares (CH-O-FML 5602); arroyo cruzando el camino Entre El Quemado y Monte Alto, San Pedro, 28/VIII/1968, 5 ejemplares, col: J. C. Viera, V. P. Ruiz y P. Autino (CH-O-FML 5611); curso de agua atravesando el camino en El Cuarteadero, San Pedro, 28/VIII/1968, 1 ejemplar, col: J. C. Viera, V. P. Ruiz y P. Autino (CH-O-FML 5725); arroyo Yacuyoto, RN 34, General José de San Martín, 19/VI/1971, 1 ejemplar, col: V. P. Ruiz (CH-O-FML 6017); arroyito sobre RN 34, El Socorro, Ledesma, 28/VI/1971, 1 ejemplar, col: V. P. Ruiz (CH-O-FML 6114); algunos ejemplares (CH-O-FML 6115).

^CATAMARCA: río El Mojón, El Alto, 01/X/1976, 2 ejemplares (uno con espermatóforo), col: T. Fasola (CH-O-FML 6849).

*CORRIENTES: charco pantanoso en el camino entre Corrientes e Ita ibaté (Aprox. 70-73 km de Corrientes), 29/XII/1949, muchos ejemplares, col: K. Gavrilov y Z. Tomsic (CH-O-FML 1380) (CH-O-FML 1380a); en el barro entre las raíces en campos de Arroz, Capital, 29/V/1950, col: W. A. D’ Angelo (CH-O-FML 1510).

${ }^{\star}$ CHACO: charco temporario en Colonia Benítez, Primero de Mayo, 10/XII/1948, varios ejemplares, col: K. Gavrilov (limo enviado por R. Golbach) (CH-O-FML 834); arroyo Margarita Belén, R11, cerca de Resistencia en dirección a Formosa, $1^{\circ}$ 
de Mayo, 27/X/1973, algunos ejemplares, col: V. P. Ruiz (CH-O-FML 6398); orilla de una represa, R11, pasando al norte algunos km el arroyo Margarita Belén, $1^{\circ}$ de Mayo, 27/X/1973, 3 ejemplares, col: V. P. Ruiz (CH-O-FML 6407); acequia R11 altura km 1061, $1^{\circ}$ de Mayo, 10/XI/1973, pocos ejemplares col: V. P. Ruiz (CH-O-FML 6472); algunos ejemplares (CH-O-FML 6473) (CH-O-FML 6474).

^FORMOSA: Clorinda, sobre el río Pilcomayo, pasando el puesto de Gendarmería, al costado de un puente, 02/XI/1973, algunos ejemplares, col: V. P. Ruiz (CHO-FML 6437); Arroyo Pucu, R11 cerca del aeropuerto de Formosa, Pilcomayo, 06/XI/1973, algunos ejemplares, col: V. P. Ruiz (CH-O-FML 6450).

$\star \star$ SANTA FE: acequia sobre R11 pasando Calchaquí hacia el norte, Capital, 23/X/1973, algunos ejemplares, col: V. P. Ruiz (CH-O-FML 6356); orilla de la acequia en Margarita, pasando algunos km esta localidad por R11, Vera, 23/X/1973, 2 ejemplares, col: V. P. Ruiz (CH-O-FML 6360); Arroyo Espin en Margarita, pasando algunos km esta localidad por R11, Vera, 24/X/1973, 5 ejemplares, col: V. P. Ruiz (CH-O-FML 6365), algunos ejemplares (CH-O-FML 6368).

$\star \star B U E N O S$ AIRES: margen del canal del Club Almirante Brown, Isla Maciel, 06/VI/1948, varios ejemplares, col: K. Gavrilov (CH-O-FML 599); costa del río Paraná en Punta Lara (cerca de La Plata), 29/V/1951, varios ejemplares, col: K. Gavrilov (CH-O-FML 2129).

*MENDOZA: pantano el lado del camino entre Cacheuta y Potrerillos, Luján de Cuyo, 02/V/1949, muchos ejemplares, col: K. Gavrilov (CH-O-FML 1129)

$\star \star$ ENTRE RÍOS: Arroyo Chico, camino de Puerto Constanza y Gualeguaychú, 01/VI/1951, ejemplares, col: K. Gavrilov (CH-O-FML 2242); arroyo Teodoro Ríos en Viale, 03/VI/1951, 3 ejemplares, col: K. Gavrilov (CH-O-FML 2284).

\section{Género Chaetogaster von Baer, 1827 Chaetogaster sp.}

Distribución y hábitat.- De amplia distribución, se encuentra en América del Norte, Europa, África, Asia y en Sudamérica en Bolivia, Perú, Brasil, Uruguay, Colombia, Surinam. En Argentina se encuentra en las provincias de San Juan, Buenos Aires, Santa Fé, Entre Ríos, Corrientes, Misiones, Santiago del Estero y en este trabajo se amplía la distribución a la provincia de Jujuy. Se encuentran en aguas estancadas o corrientes dulces o saladas. Como no son buenos nadadores viven sobre sustratos sólidos como rocas, hojas de algunas plantas, etc. Las especies de este género son depredadoras cazando y consumiendo pequeños organismos como rotíferos y cladóceros (Strait, 1977)

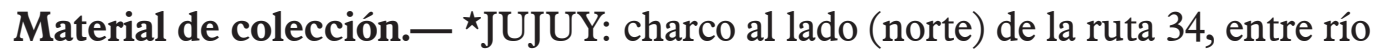
Zora y arroyo Los Berros, Calilegua, 20/XII/1966, varios ejemplares, col: K. Gavrilov, J. L. Viera, V. T. Ruiz y T. Fasola (CH-O-FML 5183). 
Género Dero Oken, 1815

Dero obtusa D'Udekem, 1855

Distribución y hábitat. - Esta especie presenta amplia distribución, América Central, y del Norte, Europa, África y Asia, en Sudamérica la encontramos en Bolivia, Surinam, Brasil, Uruguay, Venezuela y en Argentina en las provincias de Buenos Aires, Santa Fe, Corrientes, Córdoba, Entre Ríos y Santiago del Estero. En el presente trabajo se amplía la distribución de esta especie a las provincias de Tucumán, Salta y Jujuy. Esta especie tubícola se encuentra generalmente en ambientes lénticos, adheridos en algunas ocasiones, en el envés de las hojas de plantas acuáticas dentro de tubos transparentes (Marcus, 1943; Di Persia, 1976).

Material de colección.— ${ }^{\star} T U C U M A ́ N$ : acequia en el camino entre Trancas y Rosario de la Frontera, $7 \mathrm{~km}$ del río Tala (límite de la provincia) y $8 \mathrm{~km}$ de Trancas, 02/VIII/1948, varios ejemplares, col: K. Gavrilov (CH-O-FML 741); Arroyo Puente de Hierro, Bella Vista, 18/IV/1952, 1 individuo, col: K. Gavrilov (CH-O-FML 2901) (CH-O-FML 2902); Arroyo El Toro, a $17 \mathrm{~km}$ por R301, Lules, 15/V/1973, 4 ejemplares, col: E. Rivas de Pantorrilla (CH-O-FML 6281); 11 ejemplares (CH-OFML 6282).

*SALTA: Laguna caballero, $3 \mathrm{~km}$ al oeste de la estación Arenal, Rosario de la Frontera, 21/VII/1968, 5 ejemplares, col: V. P. Ruiz (CH-O-FML 5496).

^JUJUY: Bañado a $1 \mathrm{~km}$ después del Arroyo Yuto, Ruta entre Caimancito y Yuto, 26/VIII/1968, 45 ejemplares, col: J. C. Viera, V. P. Ruiz y P. Autino (CH-OFML 5716).

Dero sp.

Distribución y hábitat.- Se amplía la distribución del género a las provincias de Santiago del Estero, Chaco y Formosa y se registran nuevas localidades para Tucumán, Salta, Jujuy, Córdoba, Corrientes, Entre Ríos y Buenos Aires. A este género se lo encuentra preferentemente en arroyitos, pantanos, charcas, estanques y lagunas. Generalmente se asocia a macrófitas como Cabomba sp.; Cerathophylum sp.; Scirpus sp.; Eichhornia azurea; Hydrilla verticillata; Egeria najas (Trivinho-Strixino, Correia, Sonoda, 2000; Behrend, Takeda, Gomes, Fernandes, 2012).

Material de colección.— $\star \star$ TUCUMÁN: Monteros, pantano sobre el camino, entre Acheral y Monteros, 08/VII/1947, 10/VII/1947, varios ejemplares, col: E. H. Cordero y K. Gavrilov (CH-O-FML 138) (CH-O-FML 147) (CH-O-FML 147a) (CH-O-FML 148) (CH-O-FML 149); arroyo que cruza el camino entre Bella Vista y García Fernández, 14/VII/1947, col: K. Gavrilov (CH-O-FML 163); Monteros, pantano sobre el camino, entre Acheral y Monteros, 10/VII/1947, varios ejemplares, col: K. Gavrilov (CH-O-FML 246); Monteros, pantano sobre el camino entre Acheral y Monteros, 23/IX/1947, 55 ejemplares, col: K. Gavrilov (CH-O-FML 213) (CH-O-FML 222); pantano en el km 52 del camino entre Acheral y Monteros, 24/ 
II/1948, 3 ejemplares, col: K. Gavrilov (CH-O-FML 509), 2 ejemplares, (CH-O-FML 509a) Monteros, pantano sobre el camino, entre Acheral y Monteros, 19/VII/1948, 1 ejemplar, col: K. Gavrilov (CH-O-FML 769); pantano en el km 52 del camino entre Acheral y Monteros, 07/XII/1948, 12 ejemplares, col: K. Gavrilov (CH-OFML 829), en total varios ejemplares (CH-O-FML 830) (CH-O-FML 830a) (CHO-FML 830b) (CH-O-FML 830c); pantano en el km 52 del camino entre Acheral y Monteros, 07/XII/1948, 2 ejemplares, col: K. Gavrilov y G. Paz (CH-O-FML 878), 6 ejemplares (CH-O-FML 878a); estanque en el Instituto Miguel Lillo, San Miguel de Tucumán, 23/X/1954, 2 zooides de la misma cadena, col: J. C. Viera (CH-O-FML 2033); pantano sobre el camino entre Acheral y Monteros, 12/II/1949, 3 ejemplares, col: K. Gavrilov (CH-O-FML 2373); algunos ejemplares (CH-O-FML 2374) (CH-OFML 2375); pantano en el camino entre Acheral y Monteros (52 km de Tucumán), 18/IV/1952, varios ejemplares, col: K. Gavrilov y Z. Tomsic (CH-O-FML 2916); estanque en el Jardín del Instituto Lillo, San Miguel de Tucumán, 01/X/1964, 10 ejemplares, col: E. Rivas (CH-O-FML 4964); estanque en el Jardín del Instituto Lillo, San Miguel de Tucumán, 04/X/1965, algunos ejemplares, col: K. Gavrilov y J. C. Viera (CH-O-FML 4987) (CH-O-FML 4988); pileta a la izquierda de la entrada principal cerca de la estatua del búho en Jardín del Instituto Lillo, San Miguel de Tucumán, 07/II/1966, muchos ejemplares, col: K. Gavrilov (CH-O-FML 5017); pecera de cultivo común, Barrio Kennedy, San Miguel de Tucumán, 29/X/1979, col: E. Rivas de Pantorrilla (CH-O-FML 7361).

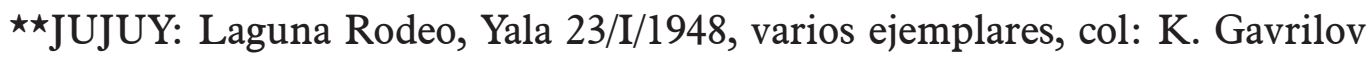
(CH-O-FML 387); pantano entre Acheral y Santa Lucía, 28/I/1948, 7 ejemplares, col: K. Gavrilov (CH-O-FML 433); río San Francisco, 5 km de la R34, entre Caimancito y Yuto, 26/VIII/1968, 159 ejemplares, col: J. C. Viera, V. P. Ruiz y P. Autino (CH-O-FML 5697).

*SANTIAGO DEL ESTERO: charco en La Banda, 15/II/1948, 3 ejemplares, col: K. Gavrilov (CH-O-FML 466); represa en El Puestito, Guasayán, 19/II/1948, 80 ejemplares, col: K. Gavrilov (CH-O-FML 477), 60 ejemplares (CH-O-FML 477a).

$\star \star S A L T A:$ pequeña acequia a orilla del camino a $10 \mathrm{~km}$ de Galpón, Metán, 17/VIII/1955, 2 ejemplares, col: J. C.Viera (CH-O-FML 2917).

${ }^{\star \star}$ CÓRDOBA: río Calabalumba, Capilla del Monte, 19/IV/1949, 4 ejemplares, col: K. Gavrilov (CH-O-FML 1176).

$\star \star$ CORRIENTES: charco pantanoso en el camino entre Corrientes e Ita Ibaté, 29/XII/1949, pocos ejemplares, col: K.Gavrilov y Z. Tomsic (CH-O-FML 1357)

${ }^{\star}$ ENTRE RÍOS: arroyito en el camino entre Viale y Villaguay, R18 (aprox. 20 km Viale), 03/VI/1951, 1 ejemplar, col: K. Gavrilov (CH-O-FML 2272), Arroyo Espinillo en el camino de Paraná a Viale, 03/VI/1951, 1 ejemplar, col: K. Gavrilov (CH-O-FML 2326).

*CHACO: charco con vegetación entre Barranqueras y Resistencia, 30/XII/1949, muchos ejemplares, col K. Gavrilov y Z. Tomsic (CH-O-FML 1400); represa al costado de la R11 cerca de Resistencia al sur, $1^{\circ}$ de Mayo, 27/X/1973, col: V. P. Ruiz (CH-O-FML 6392); Arroyo Margarita Belén R11, cerca de Resistencia en dirección a Formosa, $1^{\circ}$ de Mayo, 27/X/1973, 3 ejemplares, col: V. P. Ruiz (CH-O-FML 6402), lodo (CH-O-FML 6402-Ad). 
^FORMOSA: Arroyo San Hilario, R11, San Hilario, 06/XI/1973, varios ejemplares, col: V. P. Ruiz (CH-O-FML 6453); canal de riego que cruza la R11 en dirección a Resistencia km 1131, 10/XI/1973, varios ejemplares, col: V. P. Ruiz (CH-O-FML 6652); estero cerca del Arroyo San Hilario, San Hilario (RP5), 06/XI/1973, varios ejemplares, col: V. P. Ruiz (CH-O-FML 6654).

SANTA FE: brazo del río Paraná en el camino que se desvía en dirección a Balsa por la ruta que une Santa Fé y Helvecia, 20/V/1951, algunos ejemplares, col: K. Gavrilov (CH-O-FML 2250); Arroyo Colastiné, camino de Santa Fé en dirección a Rosario, 23/V/1951, 1 ejemplar, col: K. Gavrilov (CH-O-FML 2286).

$\star \star B U E N O S$ AIRES: arroyito tipo acequia atravesando el camino entre Buenos Aires y Zárate, R9, km 33, 31/V/1951, 1 ejemplar, col: K. Gavrilov (CH-O-FML 2253); Río Paraná cerca del club Regata en San Nicolás, 25/V/1951, 4 ejemplares, col: K. Gavrilov (CH-O-FML 2259).

Género Limnodrilus Claparède, 1862

Limnodrilus hoffmeisteri Claparède, 1862

Distribución y hábitat.- Es una especie cosmopolita, la encontramos en América Central y del Norte, Europa y Asia. En Sudamérica está presente en Chile, Perú, Colombia, Guyana, Brasil, Uruguay, Paraguay. En Argentina existen numerosos registros: Chaco, San Juan, Río Negro, Misiones, Buenos Aires, Entre Ríos, Santa Fe, Córdoba, Salta, Santiago del Estero. En el presente trabajo se amplía la distribución de esta especie a las provincias de Tucumán, Jujuy, Santiago del Estero y Catamarca y nuevas localidades para las provincias de Salta y Córdoba. Esta especie se encuentra en el sedimento de numerosos cuerpos de agua (Kennedy, 1965), constituyendo un importante eslabón de la cadena trófica en el bentos de aguas dulces y salobres (Maciorowski, Benfield, Hendricks, 1977; Woodward, Papantoniou, Edwards, Lauridsen, 2008). Por su tolerancia a los bajos contenidos de oxígeno en los cuerpos de agua es un importante bioindicador de procesos de eutrofización debido a su abundancia en sedimentos enriquecidos orgánicamente (Uzunov, Košel, Sládeèek, 1988; Burton, 1992). Constituye uno de los organismos más utilizados en estudios de ecotoxicidad y bioacumulación junto con Tubifex tubifex (Rodríguez y Reynoldson, 2011).

Material de colección.— *TUCUMÁN: arroyo pequeño en el parque, Capital, 09/V/1947, muchos ejemplares, col: K. Gravilov (CH-O-FML 86), (CH-O-FML 88); (CH-O-FML 88a); arroyo que cruza el camino entre Bella Vista y García Fernández, 14/VII/1947, 4 ejemplares, col: K. Gavrilov (CH-O-FML 178); arroyo que cruza el camino entre Bella Vista y García Fernández, 14/VII/1947, 2 ejemplares, col: K. Gavrilov, (CH-O-FML 179); arroyo que cruza el camino entre Bella Vista y García Fernández, 14/VII/1947, 1 ejemplar, col: K. Gavrilov, (CH-O-FML 181); 23/IX/1947, 2 ejemplares, col: K. Gavrilov, (CH-O-FML 243); 6 ejemplares, col: K. Gavrilov, (CH-O-FML 243a) Tafí del Valle, pequeño arroyo, $2000 \mathrm{msnm}, 11 / \mathrm{XII} / 1947$, varios ejemplares, col: K.Gavrilov (CH-O-FML 276), Tafí del Valle, charco, 11/XII/1947, varios ejemplares, col: K.Gavrilov (CH-O-FML 283) (CH-O-FML 284); Trancas, 
Río Tala, 13/12/1947, varios ejemplares, col: N. N. Kusnezov, (CH-O-FML 287); camino entre Acheral y Tafí del Valle, km 35, pantano cerca del camino, entre 1050$1100 \mathrm{msnm}, 11 / \mathrm{XII} / 1947$, varios ejemplares sexualmente maduros, col: K. Gavrilov (CH-O-FML 296), varios ejemplares (CH-O-FML 296a); varios ejemplares con involución de los órganos genitales (CH-O-FML 296b); 1 ejemplar (CH-O-FML 297); Arroyo Tapia, Trancas, 10/I/1948, varios ejemplares, col: K. Gavrilov (CH-OFML 310); varios ejemplares (CH-O-FML 310a); 1 ejemplar (CH-O-FML 311); San Miguel de Tucumán, acequia en la calle, 13/I/1948, 2 ejemplares, col: R. Golbach (CH-O-FML 317); acequia que cruza el camino al salir del pueblo en dirección a Acheral, 28/I/1948, pocos ejemplares, col: K. Gavrilov (CH-O-FML 431); 3 ejemplares (CH-O-FML 432); Villa Padre Monti, Burruyacu, , 06/II/1948, 8 ejemplares, col: R. Golbach (CH-O-FML 439); canal de Arcadia, del dique en el río Gastona, Chicligasta, 24/II/1948, 50 ejemplares, col: K.Gavrilov (CH-O-FML 507); 20 ejemplares (CH-O-FML 507a); 19 ejemplares (CH-O-FML 507b); 20 ejemplares (CH-O-FML 507c); 21 ejemplares (CH-O-FML 507d); acequia en Km 1490 del camino entre León Roúges y Monteros, 24/II/1948, 11 ejemplares, col: K. Gavrilov (CH-O-FML 508); 7 ejemplares (CH-O-FML 508a); 4 ejemplares (CH-O-FML 508b); pantano al lado del camino entre Acheral y Tafí del Valle, km 35-37, 02/XII/1947, varios ejemplares , col: K. Gavrilov (CH-O-FML 545); camino entre Trancas y Rosario de la Frontera, km 1389, 02/VIII/1948, 6 ejemplares, col: K. Gavrilov (CH-O-FML 728); acequia en el cruce calles San Luis y Bolívar, San Miguel de Tucumán, 18/XI/1948, algunos ejemplares, col: K. Gavrilov (CH-O-FML 785); acequia en el cruce calles Amador Lucero y Crisóstomo Álvarez, San Miguel de Tucumán, 18/XI/1948, muchos ejemplares, col: K. Gavrilov (CH-O-FML 816); acequia en el cruce calles San Luis y Bolívar, San Miguel de Tucumán, 07/XII/1948, 2 ejemplares maduros sexualmente, col: K. Gavrilov (CH-O-FML 820); acequia en el cruce calles San Luis y Avenida Roca, San Miguel de Tucumán, 18/XI/1948, varios ejemplares, col: K. Gavrilov (CHO-FML 823); arroyito, 2 km en dirección a Padilla (R 38), Bella Vista, 01/IX/1949, 1 ejemplar, col: K. Gavrilov y Z. Tomsic (CH-O-FML 1309); acequia, en el cruce de las calles Amador Lucero y Crisóstomo Álvarez, San Miguel de Tucumán, 18/XI/1948, 1 ejemplar, col: K. Gavrilov (CH-O-FML 2371); orilla de una vertiente en Villa Tafí del Valle, 19/I/1951, varios ejemplares, col: A. Pisano y J. Greco (CH-O-FML 2379); en agua y lodo en La Cocha, 15/VIII/1955, varios ejemplares, col: J. A. Correa (CHO-FML 2501); orilla de una vertiente en Villa Tafí del Valle, 11/XI/1947, algunos ejemplares, col: K. Gavrilov y R. Golbach (CH-O-FML 2735); orilla de una vertiente Villa Tafí del Valle, 22/III/1951, 5 ejemplares, col: K. Gavrilov y R. Golbach (CH-OFML 2736); orilla de una vertiente en Villa Tafí del Valle, 16/III/1951, 10 ejemplares , col: K. Gavrilov y R. Golbach (CH-O-FML 2743); orilla de una vertiente en Villa Tafí del Valle, 29/V/1952, 2 ejemplares (comienzo de maduración-mezclados), col: K. Gavrilov y R. Golbach (CH-O-FML 2744); orilla de una vertiente en Villa Tafí del Valle, 01/III/1951, 10 ejemplares, col: K. Gavrilov y R. Golbach (CH-O-FML 2746); orilla de una vertiente en Villa Tafí del Valle, 29/V/1952, 2 ejemplares al comienzo de la maduración (mezclados), col: K. Gavrilov y R. Golbach (CH-O-FML 2750); orilla de una vertiente en Villa Tafí del Valle, 21/V/1951, 1 ejemplar, col: K. Gavrilov y R. Golbach (CH-O-FML 2752); orilla de una vertiente en Villa Tafí del Valle, 22/ 
VI/1952, 1 ejemplar, col: K. Gavrilov y R. Golbach (CH-O-FML 2755); orilla de una vertiente Villa Tafí del Valle, 22/VII/1952, 1 ejemplar, col: K. Gavrilov y R. Golbach (CH-O-FML 2763); orilla de una vertiente en Villa Tafi del Valle, 15/III/1951, 10 ejemplares, col: K. Gavrilov y R. Golbach (CH-O-FML 2767); orilla de una vertiente en Villa Tafi del Valle, 01/06/1952,1 ejemplar, Col: K. Gavrilov y R. Golbach (CHO-FML 2774); orilla de una vertiente en Villa Tafi del Valle,14/03/1951, 1 ejemplar, Col:K. Gavrilov y R. Golbach (CH-O-FML 2778); orilla de una vertiente en Villa Tafi del Valle, 23/V/1952, 1 ejemplar, col: K. Gavrilov y R. Golbach (CH-O-FML 2783); orilla de una vertiente en Villa Tafi del Valle, 21/V/1952, 1 ejemplar, col: K. Gavrilov y R. Golbach (CH-O-FML 2788); Arroyo del Toro entre García Fernández y Bella Vista, 18/IV/1952, 1 ejemplar maduro y 2 inmaduros, col: K.Gavrilov $(\mathrm{CH}-$ O-FML 2911); 3 ejemplares (CH-O-FML 2912); Arroyo San Francisco, R9, Km 1376-1377, Trancas, 19/XII/1966, 4 ejemplares maduros +6 ejemplares inmaduros, col: K. Gavrilov, J. L. Viera, V. T. Ruiz y T. Fasola (CH-O-FML 5129); margen del río Calera, Burruyacu, 01/X/1968, 14 ejemplares, col: T. Fasola (CH-O-FML 5526); 21 ejemplares (CH-O-FML 5527); 15 ejemplares todos parasitados (CH-O-FML 5528); acequia en la estancia del Dr. Vera, $16 \mathrm{~km}$ al este de la ciudad de Trancas, 20/X/1968, 1 ejemplar maduro + 2 inmaduros, col: T. Fasola (CH-O-FML 5536); jardín del Instituto Miguel Lillo, pileta frente al palomar, San Miguel de Tucumán, 18/XI/1969, 13 ejemplares, col: K. Gavrilov y V.P. Ruiz (CH-O-FML 5774); acequia que atraviesa la casa del Sr. Rueda en San Pedro de Colalao, Trancas, 29/VIII/1969, 6 ejemplares, col: T. Fasola (CH-O-FML 5777); Arroyo Manantial, Lules, 23/IV/1970, 7 ejemplares, col: V. P. Ruiz (CH-O-FML 5903); varios ejemplares (CH-O-FML 5904) ( CH-O-FML 5905); charco a orillas del Arroyo Manantial, Lules, 20/V/1971, 2 ejemplares, col: V. P. Ruiz (CH-O-FML 5959); Arroyo El Toro, a $17 \mathrm{~km}$ por R 301, Lules, 03/X/1973, 2 ejemplares, col: E. Rivas de Pantorrilla (CH-O-FML 6280); pantano en el camino entre Acheral y Tafí del Valle, aprox. Km 35-37, Tafí del Valle, 11/XII/1947, varios ejemplares, col: K. Gavrilov (CH-O-FML 6717); Jardín del Instituto Miguel Lillo, pileta frente a la tumba del Dr. Miguel Lillo, San Miguel de Tucumán, 17/XII/1975, 9 ejemplares, col: M. T Villalba de Gavrilov y E. A. Rivas de Pantorrilla (CH-O-FML 6734), varios ejemplares (CH-O-FML 6788); jardín del Instituto Miguel Lillo, pileta cerca de la reproducción de dinosaurios, San Miguel de Tucumán, 27/V/1976, 11 ejemplares, col: $M$. T. Villalba de Gavrilov y E. Rivas de Pantorrilla (CH-O-FML 6789); jardín del Instituto Miguel Lillo, pileta frente a la tumba del Dr. M. Lillo, San Miguel de Tucumán, 27/XII/1976, 3 ejemplares col: M. T. Villalba de Gavrilov (CH-O-FML 6853).

$\star \star$ CÓRDOBA: Río Primero, Villa Warcalde, 26/II/1967, 5 ejemplares, col: K. Gavrilov (CH-O-FML 5102) (CH-O-FML 5103).

^JUJUY: acequia que cruza el camino en Humahuaca, 30/VII/1948, 25 ejemplares, col: K. Gavrilov (CH-O-FML 729); 1 ejemplar (CH-O-FML 729a); 2 ejemplares (CH-O-FML 729b); muchos ejemplares (CH-O-FML 730) (CH-O-FML 730a) (CHO-FML 730b); Río Zapla, Palpalá, 31/VII/1948, muchos ejemplares, col: K.Gavrilov, (CH-O-FML 733) (CH-O-FML 733a) (CH-O-FML 733b); acequia cruzando el camino cerca de Palos Blancos, 01/VIII/1948, algunos ejemplares, col: K.Gavrilov (CHO-FML 743); acequia de tipo arroyo en Barro Negro, San Pedro, 01/VIII/1948, varios 
ejemplares, col: K. Gavrilov (CH-O-FML 744) (CH-O-FML 744a); acequia que cruza el camino entre Jujuy y San Pedro de Jujuy, km 16, 31/VII/1948, varios ejemplares, col: K. Gavrilov, (CH-O-FML 746); 2 ejemplares (CH-O-FML 747); acequia cerca de Tilcara, 30/VII/1948, algunos ejemplares, col: K. Gavrilov (CH-O-FML 748); arroyo al lado izquierdo del camino, Maimará, 29/VII/1948, muchos ejemplares, col: K. Gavrilov (CH-O-FML 749); entre Calilegua y Río Zora, Ledesma, 19/XII/1966, 1 ejemplar, col: K. Gavrilov ,J. L. Viera, V. T. Ruiz y T. Fasola (CH-O-FML 5158); muchos ejemplares ( CH-O-FML 5159) (CH-O-FML 5160); Arroyo Los Berros cruzando la ruta 34, Calilegua, 20/XII/1966, 2 ejemplares col: K. Gavrilov , J. L. Viera, V.T. Ruiz y T. Fasola (CH-O-FML 5169); acequia al lado del camino hacia Jaramillo cruzando la ruta 34 entre Calilegua y el Río Zora, Calilegua, 20/XII/1966, muchos ejemplares, col: K. Gavrilov , J. L. Viera, V.T. Ruiz y T. Fasola (CH-O-FML 5196) (CH-O-FML 5197) (CH-O-FML 5198) (CH-O-FML 5199), lodo de una zanjita de desagüe de un grifo, entre las plantas, Barrio Ortiz, R9, San Salvador de Jujuy, 25/ VIII/1967, muchos ejemplares, col: K. Gavrilov, J. L. Viera, V. T. Ruiz y T. Fasola (CH-O-FML 5278); margen de una zanjita de desagüe de un grifo, entre las plantas Barrio Ortiz, R9, San Salvador de Jujuy, 25/VIII/1967, 5 ejemplares, col: K. Gavrilov , J. L. Viera, V. T. Ruiz y T. Fasola (CH-O-FML 5286); Laguna Rodeo, 2100 msnm, 3ra laguna en su margen nordeste, Yala, 25/VIII/1967, muchos ejemplares, col: K. Gavrilov , J. L. Viera, V. T. Ruiz y T. Fasola (CH-O-FML 5299) (CH-O-FML 5299a) (CH-O-FML 5302) (CH-O-FML 5303) (CH-O-FML 5304) (CH-O-FML 5305), 1 ejemplar ( CH-O-FML 5303a); Laguna Comedero, $2100 \mathrm{msnm}$, la 1ra laguna, Yala, 25/VIII/1967, pocos ejemplares, col: K. Gavrilov , J. C.Viera, V. P. Ruiz y T. Fasola (CH-O-FML 5324); arroyito-cascada que cruza la ruta en el camino de entrada a las Lagunas de Yala, 25/VIII/1967, pocos ejemplares, col.: K. Gavrilov, J. C. Viera, V. P. Ruiz y T. Fasola (CH-O-FML 5335); charco al lado del camino de entrada a las Lagunas de Yala, $1 \mathrm{~km}$ desde la ruta 9, 25/VIII/1967, 4 ejemplares más grandes + 2 juveniles, col: K. Gavrilov, J. C. Viera, V. P. Ruiz y T. Fasola (CH-O-FML 5349); arroyito a lo largo del camino desde S. S. de Jujuy a Termas de Reyes, $4 \mathrm{~km}$ desde el motel Huaico, 26/VIII/1967, 1 ejemplar maduro+ 6 ejemplares inmaduros, col: K. Gavrilov, J. C. Viera, V. P. Ruiz y T. Fasola (CH-O-FML 5355); 1 ejemplar parasitado con un cestodo (CH-O-FML 5356); arroyito al lado del camino en el camino de entrada a Termas de Reyes, 6 km desde ruta 9, 26/VIII/1967, muchos ejemplares, col: K. Gavrilov, J. C. Viera, V. P. Ruiz y T. Fasola (CH-O-FML 5359) (CH-O-FML 5360); 3 ejemplares jóvenes (CH-O-FML 5361); acequia al lado del camino, a 600 o $700 \mathrm{~m}$ después del río Los Alisos, camino desde S. S. Jujuy a El Carmen, 26/VIII/1967, 6 ejemplares, col: K. Gavrilov, J. C. Viera, V. P. Ruiz y T. Fasola (CH-O-FML 5381); charco a la entrada de la oficina de Agua y Energía del Dique La Ciénaga, km 1680, El Carmen, 26/VIII/1967, 2 ejemplares, col: K. Gavrilov, J. C. Viera, V. P. Ruiz y T. Fasola (CH-O-FML 5407); Dique La Ciénaga (R9), km 1670, El Carmen, 26/VIII/1967, muchos ejemplares, col: K. Gavrilov, J. C. Viera, V. P. Ruiz y T. Fasola (CH-O-FML 5408) (CH-O-FML 5411) (CH-O-FML 5412); algunos ejemplares (CH-O-FML 5409) (CH-O-FML 5413); pocos ejemplares (CH-O-FML 5414); margen del dique La Ciénaga (R9), km 1670, El Carmen, 26/08/1967, 6 ejemplares, col: K. Gavrilov , J. C. Viera, V. P. Ruiz y T. Fasola (CH-O-FML 5419); 1 ejemplar (CH-O-FML 5420); 
acequia cruzando el camino en San Juancito (4 km antes después del Cuarteadero), El Carmen, 28/VIII/1968, 1 ejemplar parasitado, col: J. C. Viera, V. P. Ruiz y P. Autino (CH-O-FML 5594); varios ejemplares (CH-O-FML 5595); 1 ejemplar parasitado (CH-O-FML 5603); 6 ejemplares (CH-O-FML 5604); 14 ejemplares (CH-O-FML 5605); acequia a $10 \mathrm{~km}$ de la salida de General San Martin a Fraile Pintado, Ledesma, 28/VIII/1968, 5 ejemplares, col: J. C. Viera, V. P. Ruiz y P. Autino (CH-O-FML 5618); muchos ejemplares (CH-O-FML 5619); acequia al borde del camino $400 \mathrm{~m}$ antes de la población del El Milagro (entre San Pedro de Jujuy y Güemes), 28/VIII/1968, 1 ejemplar, Col: J. C. Viera, V. P. Ruiz y P. Autino (CH-O-FML 5648); acequia al lado de un cañaveral, 2 km después de Fraile Pintado, 28/VIII/1968, 13 ejemplares, col: J. C. Viera, V. P. Ruiz y P. Autino (CH-O-FML 5662); pantano (totoral) al lado del camino entre Fraile Pintado y Chalican (km 1518), 28/VIII/1968, varios ejemplares, col: J. C. Viera, V. P. Ruiz y P. Autino (CH-O-FML 5671); muchos ejemplares (CH-O-FML 5672); Río Grande, debajo del puente a San Pedro, 28/VIII/1968, 18 ejemplares; col: J. C. Viera, V. P. Ruiz y P. Autino (CH-O-FML 5686), varios ejemplares (CH-O-FML 5687); 3 ejemplares (CH-O-FML 5688); 1 ejemplar (CH-O-FML 5690); curso de agua atravesando el camino en El Cuarteadero, 28/VIII/1968, 3 ejemplares, col: J. C. Viera, V. P. Ruiz y P. Autino (CH-O-FML 5722); 7 ejemplares (CH-O-FML 5723); 2 ejemplares (CH-O-FML 5724); Arroyo Yacuyoto, R34, General José de San Martín, 19/VI/1971, 5 ejemplares, col: V. P. Ruiz (CH-O-FML 6019), varios ejemplares (CHO-FML 6020); 3 ejemplares (CH-O-FML 6022); 1 ejemplar (CH-O-FML 6023); arroyito sobre RN 34, El Socorro, Ledesma, 28/VI/1971, 3 ejemplares parasitados, col: V. P. Ruiz (CH-O-FML 6118); varios ejemplares (CH-O-FML 6122); ejemplares con parásitos (CH-O-FML 6123).

$\star \star$ SALTA: arroyo en Angastaco, 22/VII/1948, varios ejemplares, col: K.Gavrilov, (CH-O-FML 763); Laguna Arenales a $100 \mathrm{~m}$ de la ruta 55, Apolinario Saravia, km 29, 19/XII/1966, varios ejemplares, col: K. Gavrilov ,J. L. Viera, V. T. Ruiz y T. Fasola (CH-O-FML 5135); muchos ejemplares (CH-O-FML 5136); arroyo al lado del camino en La Calderilla, (Por la ruta 9, entre Cornisa y Salta, cerca del cartel indicador Mojotoro 16, Güemes 42), 26/VIII/1967, 1 ejemplar, col: K. Gavrilov , J. C. Viera, V. P. Ruiz y T. Fasola (CH-O-FML 5436); Laguna Caballero, $3 \mathrm{~km}$ al oeste de la estación Arenal, Rosario de la Frontera, 21/VII/1968, muchos ejemplares, col: V. P. Ruiz (CH-O-FML 5492) (CH-O-FML 5493); lodo de una acequia en el camino de Güemes a Laguna Cabeza de Buey, a 3 km de Güemes, 28/VIII/1968, varios ejemplares, col: J. C. Viera, V. P. Ruiz y P. Autino (CH-O-FML 5629), 5 ejemplares (CH-O-FML 5630); restos (principalmente segmentos posteriores) (CH-O-FML 5630a); 1 ejemplar anormal (CH-O-FML 5631); lagunita $1 \mathrm{~km}$ antes de la entrada a Aguaray, R 34, 27/VIII/1968, 3 ejemplares, col: J. C. Viera, V. P. Ruiz y P. Autino (CH-O-FML 5668), 5 ejemplares (CH-O-FML 5669); ler curso de agua después del río Bermejo, Oran, 26/VIII/1968, varios ejemplares, col: J. C. Viera, V. P. Ruiz y P. Autino (CH-O-FML 5713) (CH-O-FML 5714); arroyo 5 en Pocitos (Salvador Mazza), 18/VI/1971, algunos ejemplares, col: V. P. Ruiz (CH-O-FML 5980); 3 km antes de El Chorro, costado del camino, Pocitos (Salvador Mazza), 18/VI/1971, 2 ejemplares, col: V. P. Ruiz (CH-O-FML 5999); pocos ejemplares (CH-O-FML 6000); arroyito pasando $1 \mathrm{~km}$ del arroyo Agua Linda, Oran, 23/VI/1971, 1 ejemplar, col: V. P. Ruiz 
(CH-O-FML 6042) (CH-O-FML 6045) (CH-O-FML 6046); desagüe al costado de la ruta, Agua Blanca, Oran, 22/VI/1971, varios ejemplares, col: V. P. Ruiz (CH-OFML 6054) (CH-O-FML 6055); arroyito en Urundel (Km 1592), Oran, 28/VI/1971, 3 ejemplares col: V. P. Ruiz (CH-O-F ML 6099); margen de arroyo en Metán (4 km pasando Metán), 28/VI/1971, varios ejemplares, col: V. P. Ruiz (CH-O-FML 6128); arroyo pasando el puesto policial, El Tala, 28/06/1971, algunos ejemplares, col: V. P. Ruiz (CH-O-FML 6139); 3 ejemplares (CH-O-FML 6140), algunos ejemplares (CH-O-FML 6141); acequia al costado de la ruta en Tabacal, Oran, 28/VI/1971, 1 ejemplar, col: V. P. Ruiz (CH-O-FML 6846).

*SANTIAGO DEL ESTERO: Río Dulce, Termas de Río Hondo 15/II/1948, 3 ejemplares, col: R. Golbach (CH-O-FML 442).

^CATAMARCA: acequia de la Plaza Olmos y Aguilera, Belén, 09/V/1949, 1 ejemplar, col: K. Gavrilov (CH-O-FML 1248); arroyo cruzando el camino cerca del indicador km 71 en Las Estancias, 10/V/1949, pocos ejemplares, col: K. Gavrilov (CH-O-FML 1249); río El Mojón, El Alto, 01/X/1976, algunos ejemplares, col: T. Fasola (CH-O-FML 6847); varios ejemplares (CH-O-FML 6848).

BUENOS AIRES: 2do Arroyo (contando desde Zárate) en el camino de ZárateBuenos Aires, 31/V/1951, 7 ejemplares, col: K. Gavrilov (CH-O-FML 2197).

ENTRE RÍOS: Arroyo Las Tunas, Camino de Paraná a Viale, R 18, 03/VI/1951, 1 ejemplar, col: K. Gavrilov (CH-O-FML 2897); 1 ejemplar (CH-O-FML 2898).

URUGUAY, Montevideo, arroyo Miguelete (Prado), 02/VI/1947, varios ejemplares, col: K. Gravilov y P. Ferrerira Berutti (CH-O-FML 125).

\section{Limnodrilus claparedianus Ratzel, 1868}

Distribución y hábitat.- De amplia distribución, a esta especie la encontramos en América del Norte, Europa y Asia. En Sudamérica en Colombia, Uruguay y en Argentina en las siguientes provincias: San Juan, Buenos Aires, Salta y Jujuy. En el presente trabajo se indican nuevos registros de esta especie para las provincias de Salta y Jujuy. Habita sedimentos con alto contenido de materia y polución orgánica además de bajo contenido de oxígeno disuelto (Rodríguez Capítulo, Ocon; Tangorra, Paggi, Cortelezzi, Spaccesi, 2003; Rodríguez y Reynoldson, 2011)

Material de colección.— ${ }^{\star}$ JUJUY: acequia de tipo arroyo en Barro Negro, San Pedro, 01/VIII/1948, 1 ejemplar, col: K. Gavrilov (CH-O-FML 745); arroyo cruzando el camino entre El Quemado y Monte Alto, San Pedro, 28/VIII/1968, 1 ejemplar, col: J. C. Viera, V. P. Ruiz y P. Autino (CH-O-FML 5609); acequia al borde del camino $400 \mathrm{~m}$ antes de la población El Milagro (entre San Pedro de Jujuy y Güemes), 28/VIII/1968, 90 ejemplares, col: J. C. Viera, V. P. Ruiz y P. Autino (CH-O-FML 5643); 7 ejemplares (CH-O-FML 5644); 3 ejemplares (CH-O-FML 5645); restos (CH-O-FML 5646); 1 ejemplar parasitado (CH-O-FML 5647); Río Grande, debajo del puente a San Pedro, 28/VIII/1968, 1 ejemplar, col: J. C. Viera, V. P. Ruiz y P. Autino (CH-O-FML 5691); curso de agua atravesando el camino en El Cuarteadero, San Pedro, 28/VIII/1968, 1 ejemplar, col: J. C. Viera, V. P. Ruiz y P. 
Autino (CH-O-FML 5726); 3 ejemplares (CH-O-FML 5727); arroyito en Chalican, Ledesma, 28/VI/1971, col: V. P. Ruiz (CH-O-FML 6103).

$\star \star$ SALTA: acequia al costado del camino $3 \mathrm{~km}$ después de Güemes hacia Tucumán, 28/VIII/1968, 3 ejemplares, col: J. C. Viera, V. P. Ruiz y P. Autino (CH-O-FML 5581) (CH-O-FML 5582).

\section{Limnodrilus udekemianus Claparède, 1862}

Distribución y hábitat.- Es una especie cosmopolita, en Sudamérica se encuentra en Chile, Perú, Colombia, Venezuela, Brasil y en Argentina en las siguientes provincias: San Juan, Buenos Aires, Santa Fe, Entre Ríos, Córdoba, Tucumán y en la Patagonia. En el presente trabajo se amplía la distribución de la especie a la provincia de Jujuy y se registran nuevas localidades para Tucumán y Buenos Aires. Habita preferentemente en fondos limo-arcillosos (Blettler y Marchese, 2005) en aguas con contaminación orgánica, pero también podemos encontrarla menos frecuentemente en hábitats oligotróficos (Klemm, 1985, Zeybek, Pahin, Yýldýz, 2018).

Material de colección.— $\star \star T U C U M A ́ N:$ acequia en el cruce calles San Luis y Bolívar, San Miguel de Tucumán, 18/XI/1948, muchos ejemplares, col: K. Gavrilov (CH-O-FML 784); acequia en el cruce calles Amador Lucero y Crisóstomo Álvarez, San Miguel de Tucumán, 18/XI/1948, algunos ejemplares, col: K. Gavrilov (CH-O-FML 817); acequia en el cruce calles San Luis y Bolívar, San Miguel de Tucumán, 18/XI/1948, varios ejemplares, col: K. Gavrilov (CH-O-FML 825) (CHO-FML 825a); un ejemplar (CH-O-FML 825b); acequia en el cruce calles Amador Lucero y Crisóstomo Álvarez, San Miguel de Tucumán, 18/XI/1948, 160 ejemplares, col: G. Paz (CH-O-FML 862); Arroyo del Toro, entre Bella Vista y García Fernández, 01/IX/1949, muchos ejemplares, col: K. Gavrilov y Z. Tomsic (CH-O-FML 1338) (CH-O-FML 1338b)(CH-O-FML 1338e) (CH-O-FML 1338f), (CH-O-FML 1338g)(CH-O-FML 1338h) (CH-O-FML 1338i) (CH-O-FML 1338j) (CH-O-FML 1338k) (CH-O-FML 13381) (CH-O-FML 1338m) (CH-O-FML 1338n) (CH-O-FML 1338o) (CH-O-FML 1338q) (CH-O-FML 1338r); 3 ejemplares (CH-O-FML 1338a), 2 ejemplares (CH-O-FML 1338c); 5 ejemplares (CH-O-FML 1338d); 5 cocones (CH-O-FML 1338p); Arroyo del Toro entre Bella Vista y García Fernández, 01/ IX/1949, 1 ejemplar, col: K. Gavrilov y Z. Tomsic (CH-O-FML 1890); 03/XI/1947= 26/XI/47: junto con otros juveniles; 26/XII/48 = aislado en estado maduro, 1 ejemplar, col: K. Gavrilov (CH-O-FML 2922); Arroyo del Toro, en el lodo entre García Fernández y Bella Vista, 26/XII/48 = aislado en estado maduro, 1 ejemplar, col: K. Gavrilov (CH-O-FML 2923); Arroyo del Toro en el lodo entre García Fernández y Bella Vista, 14/VII/47=26/XII/48 = aislado en estado maduro, 1 ejemplar, col: K. Gavrilov (CH-O-FML 2924); albañal en la calle Marcos Paz al 719, San Miguel de Tucumán, 24/II/1961, varios ejemplares, col: A. R. Sampietro (CH-O-FML 3611); muchos ejemplares, (CH-O-FML 6719); muchos ejemplares (CH-O-FML 6720); muchos ejemplares (CH-O-FML 6721); muchos ejemplares (CH-O-FML 6722); muchos ejemplares (CH-O-FML 6723); varios ejemplares (CH-O-FML 6724). 
$\star \star B U E N O S$ AIRES: Ranelagh (calle 7 entre 14/15), Berazategui, 29/X/1959, 2 tubos en frasco común, col: K. Gavrilov (CH-O-FML 6757).

^JUJUY: acequia cruzando el camino en San Juancito (4 $\mathrm{km}$ antes después del Cuarteadero), El Carmen, 28/VIII/1968, varios ejemplares, col: J. C. Viera, V. P. Ruiz y P. Autino (CH-O-FML 5607a) (CH-O-FML 5607b).

\section{Limnodrilus sp.}

Distribución y hábitat. - Se amplía la distribución del género a las provincias de Mendoza y La Rioja y se registran nuevas localidades para las provincias de Jujuy, Salta, Córdoba, Catamarca, San Juan, Buenos Aires y Entre Ríos.

Material de colección.- TUCUMÁN: arroyo que cruza el camino entre Bella Vista y García Fernández, 14/VII/1947, 1 ejemplar, col: K. Gavrilov (CH-O-FML 176), muchos ejemplares (CH-O-FML 244) (CH-O-FML 244a) (CH-O-FML 244b) (CH-O-FML 244c) (CH-O-FML 244d) (CH-O-FML 244e); Trancas, Río Tala, 13/ XII/1947, 1 ejemplar, col: N. N. Kusnezov (CH-O-FML 288); acequia de tipo arroyo que cruza el camino entre Los Aguirre y García Fernández, 26/VI/1948, varios ejemplares, col: K. Gavrilov (CH-O-FML 629), varios ejemplares (CH-O-FML 629a), varios ejemplares (CH-O-FML 629b); acequia que cruza el camino entre García Fernández y Bella Vista, km 19 desde Tucumán, 23/IX/1947, varios ejemplares, col: K. Gavrilov (CH-O-FML 637); 1 ejemplar (CH-O-FML 637a); 2 ejemplares (CHO-FML 637b); canal en el camino entre Arcadia y Monteros, 20/VII/1948, varios ejemplares, col: K. Gavrilov (CH-O-FML 694); charco en el Jardín del Instituto Lillo, San Miguel de Tucumán, 21/X/1948, varios ejemplares, col: K. Gavrilov (CHO-FML 775); charco en el Jardín del Instituto Lillo, San Miguel de Tucumán,13/ XI/1948, 2 ejemplares, col: K. Gavrilov (CH-O-FML 782); acequia en calle Brígido Terán, San Miguel de Tucumán, 18/XI/1948, varios ejemplares, col: K. Gavrilov (CH-O-FML 818); pantano en el km 52 en el camino entre Acheral y Monteros, Monteros, 07/XII/1948, 7 ejemplares, col: K. Gavrilov (CH-O-FML 827); acequia en Acheral, (entrada desde Monteros), 07/XII/1948, varios ejemplares, col: K. Gavrilov (CH-O-FML 842); desagüe en el Jardín del Instituto Lillo, San Miguel de Tucumán, 31/I/1949, algunos ejemplares, col: K. Gavrilov y G. Paz ( CH-O-FML 860) (CHO-FML 860a); desagüe en el Jardín del Instituto Lillo, San Miguel de Tucumán, 31/I/1949, algunos ejemplares, col: G. Paz (CH-O-FML 864); acequia en el cruce calles Amador Lucero y Crisóstomo Álvarez, San Miguel de Tucumán, 24/XII/1948, algunos ejemplares, col: K. Gavrilov (CH-O-FML 865); acequia en el cruce calles San Luis y Bolívar, San Miguel de Tucumán, 23/XII/1948, varios ejemplares, col: K. Gavrilov y G. Paz (CH-O-FML 866) (CH-O-FML 866a); acequia en calle San Luis, San Miguel de Tucumán, 15/II/1949, 2 ejemplares, col: G. Paz (CH-O-FML 867); pantano en el km 52 del camino entre Acheral y Monteros, 07/XII/1948, en total varios ejemplares, col: K. Gavrilov (CH-O-FML 876)(CH-O-FML 876 ${ }^{\mathrm{a}}$ ) (CH-OFML 1117); acequia cerca de Bella Vista, a $22 \mathrm{~km}$ de Tucumán, 01/IX/1954, muchos ejemplares, col: K. Gavrilov y Z. Tomsic (CH-O-FML 1874); Arroyo del Toro entre 
Bella Vista y García Fernández, 06/IX/1954, 2 ejemplares, col: K. Gavrilov y J. C. Viera (CH-O-FML 2668); estanque en el Jardín del Instituto Lillo, San Miguel de Tucumán, 01/X/1964, 3 ejemplares, col: E. Rivas (CH-O-FML 4966); acequia en la estancia del Dr. Vera, $16 \mathrm{~km}$ al este de la ciudad de Trancas, 20/X/1968, 8 ejemplares col: T. Fasola (CH-O-FML 5533) (CH-O-FML 5534) (CH-O-FML 5535); arroyo Agua Chiquita, rio Loro, Trancas, 29/VIII/1970, 5 ejemplares, col: V. P. Ruiz (CH-OFML 5920); Finca Tulio, región de Bella Vista y Acheral, camino entre Bella Vista y Famaillá, Simoca, 18/IV/1952, col: K. Gavrilov (CH-O-FML 6780).

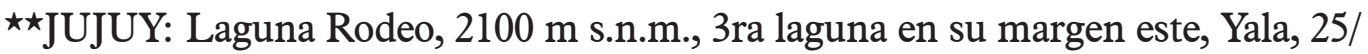
VIII/1967, 1 ejemplar juvenil muy pequeño, col: K. Gavrilov , J. C. Viera, V. P. Ruiz y T. Fasola (CH-O-FML 5313); Laguna Desaguadero, 2100 msnm, Yala, 25/VIII/1967, 2 ejemplares, col: K. Gavrilov, J. C.Viera, V. P. Ruiz y T. Fasola (CH-O-FML 5317); acequia atravesando el camino en El Quemado, San Pedro, 28/VIII/1968, 15 ejemplares, col: J. C. Viera, V. P. Ruiz y P. Autino (CH-O-FML 5578); 16 ejemplares (CH-O-FML 5579); arroyo cruzando el camino entre El Quemado y Monte Alto, San Pedro, 28/VIII/1968, 1 ejemplar col: J. C. Viera, V. P. Ruiz y P. Autino (CH-OFML 5609); 1 ejemplar aplastado, col: J. C. Viera, V. P. Ruiz y P. Autino (CH-O-FML 5610); acequia al lado de un cañaveral, 2 km después de Fraile Pintado, 28/VIII/1968, 1 ejemplar, col: J. C. Viera, V. P. Ruiz y P. Autino (CH-O-FML 5663); Río Grande, debajo del puente a San Pedro, 28/VIII/1968, varios ejemplares, col: J. C. Viera, V. P. Ruiz y P. Autino (CH-O-FML 5689); río San Francisco, $5 \mathrm{~km}$ de la R 34, entre Caimancito y Yuto, 26/VIII/1968, 1 ejemplar parasitado, col: J. C. Viera, V. P. Ruiz y P. Autino (CH-O-FML 5696); arroyo Yacuyoto, R 34, General José de San Martín, 19/VI/1971, 1 ejemplar, col: V. P. Ruiz (CH-O-FML 6018); arroyito en Chalican, Ledesma, 28/VI/1971, 4 ejemplares, col: V. P. Ruiz (CH-O-FML 6110); 2 ejemplares (CH-O-FML 6111); varios ejemplares (CH-O-FML 6112); arroyito sobre RN 34, El Socorro, Ledesma, 28/VI/1971, 1 ejemplar, col: V. P. Ruiz (CH-O-FML 6116); 2 ejemplares en desarrollo (CH-O-FML 6117); 3 ejemplares (CH-O-FML 6121).

$\star \star$ SALTA: Cachi, arroyo al lado del camino aproximadamente 7-8 km de Cachi, 23/VII/1948, muchos ejemplares, col: K. Gavrilov (CH-O-FML 674) (CH-O-FML 674a) (CH-O-FML 674b) (CH-O-FML 674c); acequia al costado del camino en Güemes, 28/VII/1948, muchos ejemplares, col: K. Gavrilov (CH-O-FML 681); acequia cerca de la finca Bella Vista en Payogasta (Camino entre Cachi a $10 \mathrm{~km}$ y Salta), 23/VII/1948, muchos ejemplares, col: K. Gavrilov (CH-O-FML 725) (CH-O-FML 725a) (CH-O-FML 725b) (CH-O-FML 725c); arroyo en Angastaco, 22/VII/1948, varios ejemplares, col: K. Gavrilov (CH-O-FML 763a); Laguna Cabeza de Buey, Güemes, 19/XII/1966, 1 ejemplar lastimado, col: K. Gavrilov ,J. C. Viera, V. T. Ruiz y T. Fasola (CH-O-FML 5148); Laguna Cabeza de Buey, Güemes, 28/VIII/1968, 2 ejemplares juveniles, col: J. C. Viera, V. P. Ruiz y P. Autino (CH-O-FML 5699); 3 $\mathrm{km}$ antes de El Chorro, costado del camino, Pocitos (Salvador Mazza), 18/VI/1971, 1 ejemplar en desarrollo, col: V. P. Ruiz (CH-O-FML 6001), 3 ejemplar (CH-O-FML 6003); desagüe al costado de la ruta, Agua Blanca, Oran, 22/VI/1971, varios ejemplares, col: V. P. Ruiz (CH-O-FML 6056).

${ }^{\star \star}$ CATAMARCA: acequia a la salida de Tinogasta, 09/V/1949, 3 ejemplares, col: K. Gavrilov (CH-O-FML 1246); pantano al lado del camino, cerca del km 64 del 
camino entre Las Estancias y Concepción, 10/V/1949, 1 ejemplar, col: K. Gavrilov (CH-O-FML 1254); rio Los Nacimientos, 1970 m, 27¹1'20” S; 66³5’37'W, Santa María, 25/XI/2003, 3 ejemplares, col: F. Romero (CH-O-FML 7943); afluente río Colorado (camino a la Hoyada por R 40), 2740 msnm, 26 35' 02"S; 66 21' 36" W, Santa María, 25/XI/2003, 7 ejemplares, Col F. Romero (CH-O-FML 7944) ^MENDOZA: Arroyo Uspallata Pozos A) y B), 32 35' 23.2'S'; 69 21' 06.9'W, Las Heras, 19/X/2015, 34 ejemplares, col: M. Peralta (CH-O-FML 7945).

$\star \star$ SAN JUAN: acequia en la calle Laprida altura 139-155, San Juan, 05/V/1949, 2 ejemplares, col: K. Gavrilov (CH-O-FML 1216); acequias al lado del camino que va a Cerro Blanco, Zonda, 05/V/1949, muchos ejemplares, col: K. Gavrilov (CH-OFML 1220) (CH-O-FML 1220a); acequias al lado del camino que va a Cerro Blanco, Zonda, 05/V/1949, varios ejemplares, col: K. Gavrilov (CH-O-FML 6760).

^LA RIOJA: acequia en Sañogasta, Chilecito, 06/V/1949, varios ejemplares, col: K. Gavrilov (CH-O-FML 1231); charco al lado de una canilla en la esquina de la Avda J. F. Quiroga y el camino para Aimogasta, Capital, 08/V/1949, muchos ejemplares, col: K. Gavrilov (CH-O-FML 1235) (CH-O-FML 1235 $)(\mathrm{CH}-\mathrm{O}-\mathrm{FML}$ 1235b).

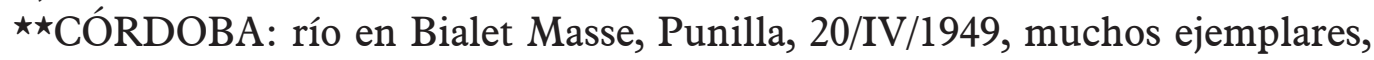
col: K. Gavrilov (CH-O-FML 1084); canal en Alto de Castro (entre Las Rosas y Villa Dolores), San Javier, 26/IV/1949, 3 ejemplares, col: K. Gavrilov (CH-O-FML 1091); Río Primero, 24/IV/1949, varios ejemplares, col: K. Gavrilov (CH-O-FML 1103) (CH-O-FML 1108), río Cosquín , 20/IV/1949, muchos ejemplares, col: K. Gavrilov (CH-O-FML 1111); acequia en Huerta Grande, Punilla, 19/IV/1949, varios ejemplares, col: K. Gavrilov (CH-O-FML 1187); Río Ceballos, Punilla, 19/IV/1949, 1 ejemplar, col: K. Gavrilov (CH-O-FML 1196).

$\star \star B U E N O S$ AIRES: Arroyo Escobar en el camino entre Buenos Aires y Zarate, 31/V/1951, 4 ejemplares, col: K. Gavrilov (CH-O-FML 2217); arroyo de tipo acequia atravesando el camino entre Buenos Aires y Zarate, R9 km 33, 31/V/1951, varios ejemplares, col: K. Gavrilov (CH-O-FML 2218), 1 ejemplar parasitado (CH-O-FML 2239); acequia del lado derecho del camino en Palomar (salida en dirección a Bella Vista), 31/V/1951, 1 ejemplar, col: K. Gavrilov (CH-O-FML 2241), algunos ejemplares parasitados con gregarinos (CH-O-FML 2249); arroyo Luna en el camino entre Rosario (pasando San Nicolás) a Buenos Aires y Zarate, R8, 25/V/1951, cantidad A/B, col: K. Gavrilov (CH-O-FML 2244), acequia en Punta Lara (cerca de La Plata), costa del río Paraná, 29/V/1951, algunos ejemplares, col: K. Gavrilov (CH-O-FML 2262); acequia del lado derecho del camino entre La Plata (Eva Perón) y Punta Lara ( a 5 km de La Plata), 29/V/1951, algunos ejemplares, col: K. Gavrilov (CH-O-FML 2265); arroyo Doña Flora en el camino entre La Plata (Eva Perón) y Punta Lara, 29/V/1951, algunos ejemplares, col: K. Gavrilov (CH-O-FML 2270).

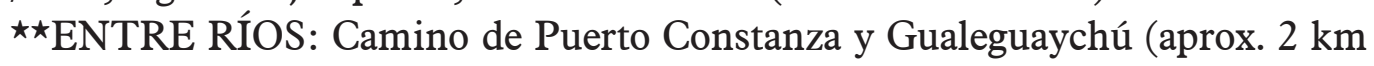
de Puerto Constanza) km 116, Islas del Ibicuy, 01/VI/1951, 6 ejemplares, col: K. Gavrilov (CH-O-FML 2220); charco pantanoso en el camino de Paraná a Viale, R18, 03/VI/1951, algunos ejemplares, col: K. Gavrilov (CH-O-FML 2266); arroyo Las Torrillas en el camino de Paraná a Viale, R18, 03/VI/1951, 3 ejemplares, col: K. Gavrilov (CH-O-FML 2271). 
Género Nais Muller, 1773

Nais sp.

Distribución y hábitat.- Cosmopolita, en Argentina hay registros en las provincias de Entre Ríos, Santa Fe, Corrientes, Santiago del Estero, Misiones, Córdoba, Buenos Aires, Patagonia y Tierra del fuego. En el presente trabajo se amplía la distribución a la provincia de Tucumán. Estos organismos se encuentran en sedimentos del fondo y tiene gran afinidad por sustratos con arena fina y pequeños fragmentos de materia orgánica (Verdonschot, 1999).

Material de colección.— ${ }^{\star}$ TUCUMÁN: pecera, Barrio Padilla, L7, San Miguel de Tucumán, 16/IX/1981, 1 ejemplar (de cultivo común), col: E. Rivas de Pantorrilla (CH-O-FML 7334); pecera de cultivo común, Barrio Padilla, San Miguel de Tucumán, 01/XI/1981, 10 ejemplares, col: E. Rivas de Pantorrilla (CH-O-FML 7337); pecera de cultivo común, Barrio Padilla, Dpto. 7, San Miguel de Tucumán, 20/XII/1980, 39 ejemplares inmaduros, col: E. Rivas de Pantorrilla (CH-O-FML 7349); pecera de cultivo común, Barrio Padilla, Dpto. 7, San Miguel de Tucumán, 1982/1983?, 75 ejemplares (cultivo común), col: E. Rivas de Pantorrilla (CH-O-FML 7358); pecera de cultivo común, Barrio Padilla, Dpto. 7, San Miguel de Tucumán, 01/XII/1982, varios ejemplares (cultivo común), col: E. Rivas de Pantorrilla (CHO-FML 7362).

Género Paranadrilus Gavrilov, 1955

Paranadrilus descolei Gavrilov, 1955

Este género y especie fue descripta por Gavrilov (1955a, 1955b, 1958) y consiste en sintipos (= Kotypen, Gavrilov 1955a), por lo tanto, ningún especimen fue designado como holotipo. Así, Rodríguez y Fend (2018), seleccionaron un especimen para ser designado Lectotipo utilizando las secciones histológicas seleccionadas por el autor para sus dibujos y descripción del sistema reproductor (CH-O-FML 2349) ParVial No.13 (Series 2349-I), 2 portaobjetos con secciones histológicas longitudinales $(10 \mu \mathrm{m})$.

Distribución y hábitat. - Esta especie se encuentra exclusivamente en Sudamérica con registros en Perú, Guyana, Brasil. En Argentina está registrada en las provincias de Entre Ríos, Santa Fe y Corrientes. En el presente trabajo se amplía la distribución a la provincia de Tucumán. En general se encuentra en ríos con profundidad y velocidad promedio, sedimentos arcillosos y baja conductividad (Marchese, 1987).

Material de colección.— `TUCUMÁN: arroyo Puente de Hierro en Bella Vista, 18/IV/1952, 1 ejemplar pequeño, col: K. Gavrilov (CH-O-FML 2910).

ENTRE RÍOS: Arroyo Malo (más cercano de Viale) camino de Paraná a Viale, R18, 03/VI/1951, algunos ejemplares, col: K. Gavrilov (CH-O-FML 2296); arroyo 
Las Tunas (aprox. 7 km de Paraná) en el camino de Paraná a Viale, R18, 03/VI/1951, algunos ejemplares, col: K. Gavrilov (CH-O-FML 2349 A-E).

\section{Género Paranadrilus sp.}

Material de colección.- SANTA FE: Lagunita (Parque Juan de Garay del Sur), 04/VI/1951, algunos ejemplares, col: K. Gavrilov (CH-O-FML 2278).

\section{Género Pristina Ehrenberg, 1828 \\ Pristina longiseta Bourne, 1891}

Distribución y hábitat.- De amplia distribución, registrada para Europa, Asia, Australia, América del Norte y Central, Hawaii. En Sudamérica se encuentra en Chile, Bolivia, Perú, Uruguay, Paraguay, Colombia, Venezuela, Surinam, Brasil. En Argentina se encuentra en las provincias de: Chaco, San Juan, Misiones, Buenos Aires, Santa Fe, Entre Ríos, Corrientes, Santiago del Estero y Tucumán. En el presente trabajo se registran nuevas localidades para las provincias del Chaco y Tucumán. Esta especie en general se encuentra asociada a plantas acuáticas como Egeria sp. y Salvinia sp. (Corbi y Trivinho-Strixino, 2002; Alves y Gorni, 2007).

Material de colección.— ${ }^{\star}$ TUCUMÁN: Jardín del Instituto Miguel Lillo, acuario natural entre las hojas de Bromeliaceae Tillandsia maxima Lillo en la base de las hojas, San Miguel de Tucumán, 08/IV/1969, algunos ejemplares, col: J. C. Viera y K. Gavrilov (CH-O-FML 5761).

${ }^{\star \star}$ CHACO: represa al costado de la R11 cerca de Resistencia al sur, La Palometa , San Fernando, 26/X/1973, 12 ejemplares color rosado con probóscide corta, col: V. P. Ruiz (CH-O-FML 6386); laguna al costado de la ruta que va a Corrientes antes del puente entre Chaco y Corrientes, 12/XI/1973, muchos ejemplares, col: V. P. Ruiz (CH-O-FML 6491) (CH-O-FML 6518); arroyito sobre R11, km 1059, Chaco, 11/XI/1973, algunos ejemplares, col: V. P. Ruiz (CH-O-FML 6657).

\section{Pristina sp.}

Distribución y hábitat. - En el presente trabajo se amplía la distribución del género a las provincias de Jujuy, Formosa y Mendoza y se registran nuevas localidades para las provincias de Tucumán, Chaco, Santa Fé, Corrientes, Entre Ríos, San Juan y La Rioja.

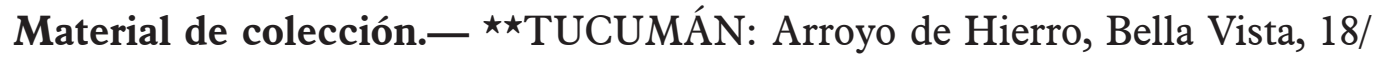
IV/1952, 4 individuos, col: K. Gavrilov (CH-O-FML 233); Charco en Los Puestos, Leales, 20/IV/1948, 5 ejemplares, col: K. Gavrilov (CH-O-FML 555), 9 ejemplares (CH-O-FML 555a); arroyo en camino de Tafí del Valle al Infiernillo, km 83, 20/ 
VII/1948, varios ejemplares, col: K. Gavrilov (CH-O-FML 721); pantano sobre el camino entre Acheral y Monteros ( $\mathrm{km} 52$ ferrocarril), 01/IX/1949, 1 ejemplar, col: K. Gavrilov (CH-O-FML 1288); Arroyo Puente de Hierro, Bella Vista, 18/IV/1952, col: K. Gavrilov (CH-O-FML 2899); lagunita detrás del ferrocarril en el camino entre Acheral y Monteros, 18/IV/1952, algunos ejemplares, col: K. Gavrilov (CHO-FML 2904).

^JUJUY: bañado a $1 \mathrm{~km}$ después del Arroyo Yuto, ruta entre Caimancito y Yuto, 26/VIII/1968, 9 ejemplares, col: J. C. Viera, V. P. Ruiz y P. Autino (CH-O-FML 5719).

^FORMOSA: represa del Cuartel de Gendarmería, Clorinda, Pilcomayo, 29/ X/1973, 5 ejemplares, col: V. P. Ruiz (CH-O-FML 6677).

${ }^{\star \star}$ CHACO: Arroyo Margarita Belén, R11, cerca de Resistencia en dirección a Formosa, $1^{\circ}$ de Mayo, 27/X/1973, 3 ejemplares, col: V. P. Ruiz (CH-O-FML 6403).

$\star \star S A N T A$ FE: río Santa Rosa, RP1, bajo el puente Garay, 20/X/1973, muchos ejemplares, col: V. P. Ruiz (CH-O-FML 6658).

$\star \star C O R R I E N T E S:$ Laguna Brava, Capital, 02/VIII/1950, 1 ejemplar, col: J. H. S. Stekhoven (CH-O-FML 2201).

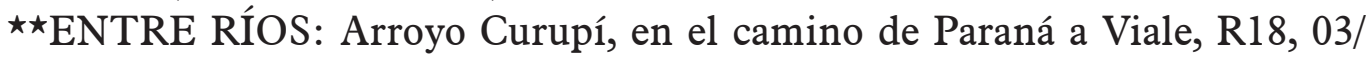
VI/1951, algunos ejemplares, col: K. Gavrilov (CH-O-FML 2258).

^MENDOZA: Arroyo Uspallata, Pozos A) y B), 32 35' 23.2'S', 69 21' 06.9'W, Las Heras, 19/X/2015, 34 ejemplares, col: M. Peralta (CH-O-FML 7946).

$\star \star$ SAN JUAN: Río Usno, hiporreico, pozo 1 (100 lts. Filtrado), 928 m s.n.m., $30^{\circ} 34^{\prime} 32,4^{\prime \prime}$ S, $67^{\circ} 34^{\prime}$ 12,1W, Valle Fértil, 04/XI/2014, 13 ejemplares, col: M. Peralta (CH-O-FML 7948).

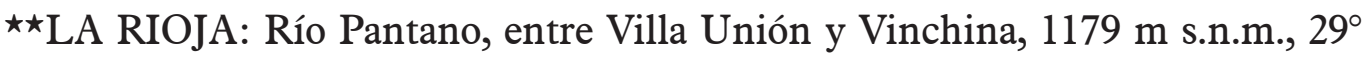
15' 51" S, 68 14' 10” W, Vinchina, 5-10/IX/2011, 83 ejemplares, col: F. Romero (CH-O-FML 7949).

\section{Género Slavina Vejdovský, 1883 \\ Slavina sawayai Marcus, 1944}

Distribución y hábitat.- Esta especie presenta distribución principalmente sudamericana, hasta el presente registrada solo para Ecuador, Brasil y Argentina en las provincias de Buenos Aires, Entre Ríos, Santa Fe. En el presente trabajo se amplía la distribución de la especie a la provincia de Jujuy. Se encuentra generalmente asociada a macrófitas acuáticas como Salvinia biloba, Egeria najas, Eichhornia crassipes (Di Persia, 1980; Gluzman, 1991, 1994).

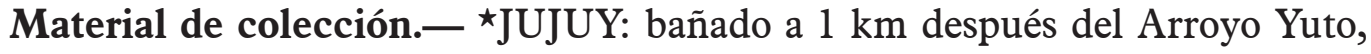
Ruta entre Caimancito y Yuto, 26/VIII/1968, 2 ejemplares, col: J. C. Viera, V. P. Ruiz y P. Autino (CH-O-FML 5718). 
Genero Tubifex Lamarck, 1816

Tubifex tubifex Muller, 1774

Distribución y hábitat.- Género cosmopolita, en Sudamérica está registrado para Paraguay, Brasil y Argentina. En esta última, en las provincias de Buenos Aires y en Córdoba en los embalses San Roque, Los Molinos y Embalse Río Tercero en aguas superficiales y con poca densidad (Bonetto, Di Persia, Maglianesi, Corigliano, 1976). En el presente trabajo se amplía la distribución de la especie a las provincias de Tucumán, San Juan, Jujuy y se registran nuevas localidades en la provincia de Buenos Aires. Es una especie poly-sapróbica (Johnson, Wiederholm, Rosenberg, 1993), típica de aguas fuertemente contaminadas por lo que es considerada bioindicador importante (Armitage, Moss, Wright, Furse, 1983). No obstante, también puede ser registrada en ambientes oligotróficos. Se encuentra habitualmente en ambientes con bajo caudal y velocidad de corriente pero con altos valores de conductividad (Marchese, 1987).

Material de colección.— *TUCUMÁN: pileta en la sección de Entomología del Jardín del Instituto Lillo, San Miguel de Tucumán, 19/I/1953, col: M. Manfrini y Z. Tomsic (CH-O-FML 2652); pileta del Departamento de Entomología en el Jardín del Instituto Lillo, San Miguel de Tucumán, 03/VI/1953, 1 ejemplar, col: K. Gavrilov y N. Paz de Tomsic (CH-O-FML 2889); Jardín del Instituto Miguel Lillo, pileta frente al palomar, San Miguel de Tucumán, 18/XI/1969, muchos ejemplares, col: K. Gavrilov y V. P. Ruiz (CH-O-FML 5775) (CH-O-FML 5776).

*SAN JUAN: acequias al lado del camino que va a Cerro Blanco, Zonda, 05/ V/1949, muchos ejemplares, col: K. Gavrilov (CH-O-FML 1221); 17 ejemplares, cultivo, (reproducción uniparental asexual) (CH-O-FML 2894), 5 individuos surgidos por vía uniparental asexual (CH-O-FML 2895).

^JUJUY: acequia cruzando el camino (orilla de la misma) en San Juancito (4 km antes de El Cuarteadero), El Carmen, 28/VIII/1968, 5 ejemplares, col: J. C. Viera, V. P. Ruiz y P. Autino (CH-O-FML 5596), 14 ejemplares (CH-O-FML 5597), 6 ejemplares (CH-O-FML 5598), varios ejemplares juveniles + fragmentos (CH-O-FML 5600).

$\star \star B U E N O S$ AIRES: acequia del lado derecho del camino al Palomar (salida en dirección a Bella Vista), 31/V/1951, 1 ejemplar, col: K. Gavrilov (CH-O-FML 2285), 2 ejemplares (CH-O-FML 2285a).

Tubifex sp.

Distribución.- Se amplía la distribución del género a la provincia de La Rioja y se registran nuevas localidades en las provincias de Jujuy y Tucumán.

Material de colección.- ${ }^{\star}$ LA RIOJA: charco al lado de una canilla en la esquina de la Avda. J. F. Quiroga y el camino para Aimogasta, Capital, 08/V/1949, muchos ejemplares, col: K. Gavrilov (CH-O-FML 1235) (CH-O-FML 1235a) (CHO-FML 1235b). 


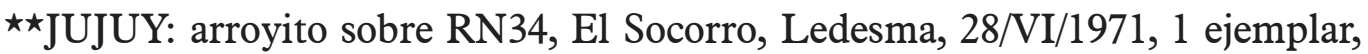
col: V. P. Ruiz (CH-O-FML 6119), 2 ejemplares (CH-O-FML 6120).

$\star \star$ TUCUMÁN: Jardín del Instituto Miguel Lillo, pileta frente al palomar, San Miguel de Tucumán, 03/VIII/1972, varios ejemplares (vistos in vivo en microscopio), col: M. T. V. de Gavrilov (CH-O-FML 6261); arroyo Bajo Hondo, calle San Martín 3800, San Miguel de Tucumán, 03/X/1973, 1 ejemplar, col: M. T. V. de Gavrilov y J. C. Viera (CH-O-FML 6270).

Familia Enchytraeidae Vejdovský, 1879

Es una familia poco estudiada de Oligochaeta con 650 especies descriptas en el mundo. Además de India y África Central, son muy comunes en los hábitats terrestres y semiacuáticos de Sudamérica subtropical en donde presentan una aparente endemicidad ya que de las 66 especies registradas, 50 son exclusivas (Coates, 1989; Christoffersen, 2009). En Sudamérica encontramos registros en Guyana, Paraguay, Bolivia, Perú, Ecuador, Colombia, Venezuela, Chile, Brasil y Argentina (Christofferson, 2009). Se encuentran en un amplio rango de hábitats acuáticos (agua dulce, salobre y marina) y terrestres (Erséus 2005; Brinkhurst y Marchese, 1991). Constituyen una parte importante de las comunidades acuáticas (Martin, Martinez-Ansemil, Pinder, Timm, Wetzel, 2008) y de las subterráneas (Giani, Sambugar, Rodríguez, Martinez-Ansemil, 2001). La mayoría de las especies son de color lechoso o transparentes, cilíndricas y pequeñas (3-4 mm), delgadas y principalmente intersticiales (Rodríguez y Reynoldson, 2011). En Brasil esta familia ha sido ampliamente citada en trabajos de ecología de agua dulce (Frizzera y Alves, 2012; Gorni y Alves, 2012; Rodrigues, Santana, Alves, 2013) mientras que en Argentina la distribución conocida hasta el presente está restringida al extremo austral (Islas Malvinas, Estrecho de Magallanes, Ushuaia) y el Nordeste (Misiones, Entre Ríos y Paraná Superior). En el presente trabajo ampliamos la distribución de la familia a las provincias de Tucumán, Jujuy, Salta, Catamarca, Córdoba, Mendoza, La Rioja y se registran nuevas localidades para Entre Ríos.

Material de colección.- ^TUCUMÁN: calle San Juan, San Miguel de Tucumán, 12/IX/1947, varios ejemplares, col: O. Budin (CH-O-FML 208); 2 ejemplares, (CH-O-FML 208a); 4 ejemplares (CH-O-FML 208b); margen del arroyo del Toro, entre Bella Vista y García Fernández, Leales, 3/IX/1947, 1 ejemplar, col: K. Gavrilov (CH-O-FML 223); 3 ejemplares (CH-O-FML 242); tierra de jardín cerca del agua en calle Córdoba 2006, San Miguel de Tucumán, 12/XI/1949, muchos ejemplares, col: R. Golbach (CH-O-FML 1295); Arroyo del Toro entre Bella Vista y García Fernández, Leales, 01/IX/1949, muchos ejemplares, col: K. Gavrilov y Z. Tomsic (CH-O-FML 1305); Quinta Agronómica, San Miguel de Tucumán, 20/IV/1951, pocos ejemplares, col: J. H. S. Stekhoven (CH-O-FML 2202); Villa Marcos Paz, Yerba Buena, 01/V/1953, col: Z. Tomsic (CH-O-FML 2365); Cerro San Javier, Yerba Buena, 07/IV/1953, col: O. Budin (CH-O-FML 2366) (CH-O-FML 2367) (CH-O-FML 2390) (CH-O-FML 2391); zanja de la pileta de Entomología del Jardín del Instituto 
Lillo, San Miguel de Tucumán, 04/VIII/1952, varios ejemplares, col: Z. Tomsic $(\mathrm{CH}-$ O-FML 2864); 30/IX/1952, pocos ejemplares (CH-O-FML 2877); tierra húmeda en la calle Paso de los Andes 328, San Miguel de Tucumán, 16/XII/1962, 1 ejemplar, col: V. P. Ruiz (CH-O-FML 3775); margen del arroyito, Jardín del Instituto Lillo, San Miguel de Tucumán, 16/III/1963, algunos ejemplares, col: K. Gavrilov (CH-O-FML 3835); mismo lugar,18/III/1963, varios ejemplares, col: V. P. Ruiz (CH-O-FML 3836); Jardín del Instituto Lillo, San Miguel de Tucumán, 27/VII/1963, 1 ejemplar, col: K. Gavrilov (CH-O-FML 3948); calle Paso de los Andes 328, San Miguel de Tucumán, 08/III/1964, varios ejemplares, col: V. P. Ruiz (CH-O-FML 4291); represa, $10 \mathrm{~km}$ al oeste de Colalao del Valle, El Pichao, Tafí del Valle, 31/VIII/1967, 3 ejemplares, col: T. Fasola (CH-O-FML 5263); margen de un charco en Ruta 38, entre San Rafael (1 km de este) y Famaillá $(8 \mathrm{~km}), 04 / \mathrm{II} / 1968,13$ ejemplares, col: K. Gavrilov e I. Gavrilov (CH-O-FML 5437); Río Tipas, San Pedro de Colalao, Trancas, 20/III/1968, 2 ejemplares, col: T. Fasola (CH-O-FML 5453); Aguada de la Sepultura en el camino de Las Arcas a la estancia de Rearte, Trancas, 26/IV/1968, 1 ejemplar, col: T. Fasola (CH-O-FML 5484); vivero de plantas de la florería Jardín Tropical, calle Los Naranjos, a la altura de la Avda Mitre 2400, San Miguel de Tucumán, 14/III/1969, 6 ejemplares, col: J. C.Viera y K. Gavrilov (CH-O-FML 5734a); 3 ejemplares (CHO-FML 5734b); Rio Barburin, estancia de Rearte, Trancas, 03/IV/1970, 1 ejemplar, col: T. Fasola (CH-O-FML 5896); margen del rio Loro, Trancas, 29/VIII/1970, 3 ejemplares, col: V. P. Ruiz (CH-O-FML 5918); Jardín del Instituto Lillo, estanque entrada edificio de Geología, San Miguel de Tucumán, 29/VIII/1970, algunos ejemplares, col: E. Rivas de Pantorrilla, J. C. Viera (CH-O-FML 5923); Horco Molle, Yerba buena, Setiembre de 1969, varios ejemplares, col M. V. Colomo (CH-O-FML 5946); 06/V/1970, 25 ejemplares (CH-O-FML 5947); pequeño arroyo en Esquina, $25 \mathrm{~km}$ al sur por R5, Leales, 28/VII/1974, 1 ejemplar incompleto, col: J. C. Nieto (CH-O-FML 6307); acequia en Quilmes (2000 o 2200 m s.n.m.), Tafí del Valle, 22/VIII/1974, 2 ejemplares, col: T. Fasola (CH-O-FML 6324), 3 ejemplares (CH-OFML 6325); Jardín del Instituto Miguel Lillo, zanja de pileta de Entomología, San Miguel de Tucumán, 04/VIII/1952, varios ejemplares, col: Z. Tomsic (CH-O-FML 6718); cerro Muñoz, 3500 m s.n.m., Tafí del Valle, 29/V/1982, algunos ejemplares, col: G. Scrocchi (CH-O-FML 6896).

^JUJUY: zanjita de desagüe en Barrio Ortiz, R9, San Salvador de Jujuy, 25/ VIII/1967, 4 ejemplares, col. K. Gavrilov, J. L. Viera, V. T. Ruiz y T. Fasola (CH-OFML 5290); arroyito-cascada que cruza la ruta, en camino de entrada a las Lagunas de Yala, 25/VIII/1967, 12 ejemplares, col: K. Gavrilov, J. C. Viera, V. P. Ruiz y T. Fasola (CH-O-FML 5348); arroyo cascada, detrás del casino, Termas de Reyes, 26/VIII/1967, 1 ejemplar, col: K. Gavrilov, J. C. Viera, V. P. Ruiz y T. Fasola (CH-OFML 5373); represa al lado del camino desde S. S. Jujuy a El Carmen, R9, km 1680, 26/VIII/1967, 1 ejemplar muy pequeño, col: K. Gavrilov, J. C. Viera, V. P. Ruiz y T. Fasola (CH-O-FML 5395); 1 ejemplar (CH-O-FML 5406).

*SALTA: margen de acequia en la Posta de Yatasto, Metán, 17/VIII/1955, col: J. C. Viera (CH-O-FML 2657); borde de una acequia en Tolombón, altura $2000 \mathrm{~m}$ s-n-m-, Cafayate, 22/VIII/1974, 6 ejemplares, col: T. Fasola (CH-O-FML 6318). 
^CATAMARCA: río Chaschuil, Chaschuil a $60 \mathrm{~km}$ de Fiambalá, Tinogasta, 18/V/1973, 14 ejemplares, col: T. Fasola (CH-O-FML 6294); varios ejemplares (CHO-FML 6294a); orilla de una acequia en Ampujaco, Belén, 26/IX/1974, algunos ejemplares, col: T. Fasola (CH-O-FML 6315).

*CÓRDOBA: Canal de Ungaro parte más cercana a la ruta Martinolli, Arguello, Capital, 9/II/1963, 2 ejemplares, col: K. Gavrilov (CH-O-FML 4819); margen del arroyito cercano a la calle Manfredi en Villa Belgrano (cerca de Arguello alt. Avda República), Capital, 15/ÎI/1965, 3 ejemplares, col: K. Gavrilov (CH-O-FML 4936); Arguello (recta Martinolli esquina Salta), Capital, 28/II-1/III//1967, muchos ejemplares, col: K. Gavrilov (CH-O-FML 5100).

^MENDOZA: Capital, 29/IX/1964, 3 ejemplares, col: R. Cei (CH-O-FML 4976).

^LA RIOJA: arroyo al lado del camino entre La Rioja y Aimogasta, 08/V/1949, 2 ejemplares, col: K. Gavrilov (CH-O-FML 1237); orilla de una vertiente en Punta del Agua, 40 km de Jaguel, Vinchina, 06/III/1970, 12 ejemplares, col: T. Fasola (CHO-FML 5878); 15 ejemplares (CH-O-FML 5879).

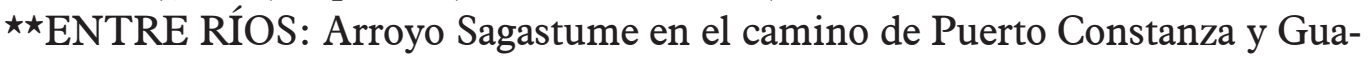
leguaychu, Islas del Ibicuy, 01/VI/1951, 2 ejemplares, col: K. Gavrilov (CH-O-FML 2189), orilla de la acequia en R14 entre Concepción del Uruguay y Gualeguaychu, km 264, 09/IX/1974, 1 ejemplar, col: V. P. Ruiz (CH-O-FML 6599).

Género Enchytraeus Henle, 1837

Enchytraeus albidus Henle, 1837

Distribución y hábitat.- También denominados "gusanos blancos» son de aspecto robusto, eurihalinos y cosmopolitas (Ghabbour, 1966) La distribución registrada en Argentina corresponde a Islas Malvinas (Stephenson, 1932), Estrecho de Magallanes, Isla Clarence, Tierra del Fuego (Ude, 1896; Černosvitov, 1934). Considerados como el alimento principal para la crianza de organismos de agua dulce y salada, como peces ornamentales y crustáceos (Fairchild, Bergman, Trushenski, 2017).

Material de colección.- URUGUAY: Arroyo Miguelete (Prado), Montevideo, 02/VI/1947, varios ejemplares, col: K. Gravilov y P. Ferrerira Berutti (CH-O-FML 111).

\section{CONCLUSIÓN}

La colección de Oligochaeta Microdrili de la Fundación Miguel Lillo no presenta tipos y solo recientemente fue nombrado el Lectotipo de Paranadrilus descolei (Rodríguez y Fend, 2018). Están representados el 31\% de los 32 géneros de Naididae registrados para Argentina y en el presente trabajo se amplía la distribución a otras provincias además de registrarse nuevas localidades para los géneros: Aulophorus; 
Bothrioneurum; Chaetogaster; Dero; Limnodrilus; Nais; Paranadrilus; Pristina; Slavina, Tubifex y para la familia Enchytraeidae. La importancia de esta colección reside no solo en el valor científico e histórico sino también en su cuidadosa organización además de preservación. Se resalta su valor como colección de referencia y como herramienta para estudios taxonómicos, biogeográficos, ecológicos, y de apoyo para la docencia. Presentando también un indiscutible valor como acervo representativo de la biodiversidad de oligoquetos en Argentina.

\section{AGRADECIMIENTOS}

Agradezco a la curadora de la Colección de Invertebrados, Dra. Marcela Peralta, por facilitarme el acceso a la misma. También agradezco a los revisores que enriquecieron este trabajo con sus sugerencias. Y finalmente a la Fundación Miguel Lillo por el constante apoyo a mi investigación.

\section{FINANCIAMIENTO}

Este trabajo fue financiado por la Fundación Miguel Lillo en el marco del proyecto Z-0089-123.

\section{PARTICIPACIÓN}

La autora ha realizado la totalidad del trabajo expuesto, con las sugerencias de la Dra. Marcela Peralta y la Dra. Geraldine Ramallo.

\section{CONFLICTO DE INTERESES}

La autora declara no tener conflictos de intereses con terceros.

\section{LITERATURA CITADA}

Aarefjord, F., Borgstrom R., Lien, 1. Milbrink, G. (1973). Oligochaetes in the bottom fauna and stomach content of trout, Salmo trutta (1.). Norwegian Journal of Zoology, 21, 281-288.

Alves, R. G.; Gorni, G. R. (2007). Naididae species (Oligochaeta) associated with submersed aquatic macrophytes in two reservoirs. São Paulo. Acta Limnologica Brasiliensia, 19 (4), 407-413.

Armitage, P. D., Moss, D., Wright, J. F., Furse, M. T. (1983). The performance of a new biological water quality score system based on macroinvertebrates over a wide range of unpolluted running-water sites. Water Research, 17 (3), 333-347. 
Baer, K. E. von (1827). Beiträge zur Kenntnis der Niedern Thiere [Tiere] III (Über Chaetotaster, Hirudo marginata und hyalina) Nova Acta phys.-med. Academiae Caesareae Leopoldino-Carolinae Germanicae Naturae Curiosorum, 13 (2), 605-615.

Beddard, F. E. (1888). Observations upon an Annelid of the Genus Aeolosoma. Proceedings of the Zoological Society of London, 213-17.

Behrend, R. D. L., Takeda, A. M., Gomes L. C., Fernandes S. E. P. (2012). Using Oligochaeta assemblages as an indicator of environmental changes. Brazilian Journal of Biology, São Carlos, 72 (4), 873-884.

Benham, W. B. (1890). An attempt to classify earthworms. Quarterly Journal of the Microscopical Society, 31, 201-315.

Blettler, M. C. M., Marchese M. R. (2005). Effects of bridge construction on the benthic invertebrates structure in the Paraná river Delta. Interciencia, 3 (2), 60-66.

Bonetto, A., Di Persia, D, Maglianesi, R., Corigliano, M. (1976). Caracteres Limnológicos de Algunos Lagos Eutróficos de Embalse de la Región Central Argentina. Ecosur, 3 (5), 47-120.

Bourne, A. G. (1891).Notes on the naidiform Oligochaeta .Quarterly Journal of Microscopical Science, 32, 335-356.

Brinkhurst, R. O. (1996). On the role of tubificid oligochaetes in relation to fish disease with special reference to the Myxozoa. Annual Review of Fish Diseases, 6, 29-40.

Brinkhurst, R. O., Cook, D. G. (1980). Aquatic Oligochaete Biology. Plenum Publisher Corporation, New York.

Brinkhurst, R. O., Wetzel, M. J. (1984). Aquatic Oligochaeta of the World: Supplement. A Catalogue of New Freshwater Species, Descriptions, and Revisions. Canadian Technical Report Hydrography and Ocean Sciences, 44, v + 101 p.

Brinkhurst, R. O., Marchese, M. R. (1991). Guía para la identificación de oligoquetos acuáticos continentales de Sud y Centro América. Colección Climax $\mathrm{N}^{\mathrm{o}}$ 6, Segunda edición. Asociación de Ciencias Naturales del Litoral. Santa Fe, Argentina.

Bunke, D. (1988). Aeolosomatidae and Potamodrilidae. En Introduction to the Study of Meiofauna (345-348). Smithsonian Institution Press, Washington, D.C.

Burton, G. A. (1992). Sediment toxicity assessment. London: Lewis Publishers, Inc.

Coates, K. A. (1989). Phylogeny and origins of Enchytraeidae (Annelida: Oligochaeta). Hydrobiologia, 180, 17"33.

Černosvitov, L. (1934). Oligochètes. Expedition Antarctique Belge Ressultats du Voyage de la Belgica 1897-99. Rapports Scientifiques Zoologie, 1934, 1"11.

Claparède, É. (1862). Recherches anatomiques sur les Oligochètes. Mémoires de la Société de Physique et d'Histoire Naturelle de Genève, 16 (2), 217-291.

Christoffersen, M. L. (2007). A catalogue of aquatic microdrile oligochaetes (Annelida: Clitellata) from South America. Acta Hydrobiologica Sinica, 31, 59-86. 
Christoffersen, M. L. (2010). Continental biodiversity of South American Oligochaetes: the importance of inventories. Acta Zoológica Mexicana, 2, 35-46.

Corbi, J. J.; Trivinho-Strixino, S. (2002). Spatial and bathymetric distribution macrobenthic fauna of the Ribeirão das Anhumas reservoir (Américo Brasiliense-SP, Brasil). Acta Limnologica Brasiliensia, 10 (1), 37-47.

d'Udekem, J. (1855). Nouvelle clasification des Annélides sétigeres abranches. Bulletins de l'Académie Royale des Sciences, des Lettres et des Beaux-Arts de Belgique, 22, 533-555.

Di Persia, D. H. (1976). Nuevas citas del género Dero S.S. (Naididae, Tubificoidea) para la Oligoquetofauna acuática Argentina. Physis, B 35, 1-7.

Dyne G. R. y Jamieson B. G. (2004) Native Earthworms of Australia II. (Megascolecidae, Acanthodrilinae). ABRS. Australian Government Department of Environment and Heritage.

Ehrenberg, Ch. G. (1828). Symbolae physicae seu Icones Descriptiones Corporum naturalium novorum aut minus cognitorum, quae ex Itineribus per Lybyam, Aegyptum, Publico institutis Sumptu Animalia evertebrata. Phytozoa. Decas, 1, 1-293.

Erséus, C. (2005). Phylogeny of oligochaetous Clitellata. Hydrobiologia, 535 (536), 357-372.

Erséus, C, Wetzel, M, Gustavsson, L. (2008). ICZN rules - a farewell to Tubificidae (Annelida, Clitellata). Zootaxa, 1744, 66-68.

Falci Theza Rodrigues, L., Jabour Vescovi Rosa, B. F., Lobo, H., Campos Divino, A., da Gama Alves, R. (2015). Diversity and Distribution of Oligochaetes in Tropical Forested Streams, Southeastern Brazil. Journal of Limnology, 74 (3), 433-443.

Fairchild, E.A., Bergman, A. M., Trushenski, J. T. (2017). Production and nutritional composition of white worms Enchytraeus albidus fed different low-cost feeds. Aquaculture, 481, 16-24.

Frizzera, G., Alves, R. (2012). The influence of taxonomic resolution of Oligochaeta on the evaluation of water quality in an urban stream in Minas Gerais, Brazil. Acta Limnologica Brasiliensia, 24, 408-416.

Gavrilov, K. (1955). Ein neuer spermathekenloser Vertreter der Tubificiden. Zoologische. Anzeiger, 155, 294-302.

Gavrilov, K. (1955b). Über die uniparentale Vermehrung von Paranadrilus. Zoological Anzeiger, 155, 302-306.

Gavrilov, K. (1958). Notas adicionales sobre Paranadrilus. Acta Zoologica Lilloana, XVI, 149-235.

Gavrilov, K. (1979). Oligochaeta. En Biota acuática de Sudamérica Austral (99-121). San Diego State University, San Diego.

Gavrilov, K. (1981). Oligochaeta. En Aquatic biota of tropical South America. Being a compilation of taxonomic bibliographies for the fauna and flora of inland waters of the tropical portion of South America. Part 2. Anarthropoda (170190).San Diego University, San Diego.

Ghabbour, S. I., (1966). The importance of oligochaetes in fish culture: a review with special reference to Lake Nasser. Progress of Fish Culture, 28, 206-215. 
Giani N., Sambugar, B., Rodríguez, P., Martinez-Ansemil, E. (2001). Oligochaetes in southern European groundwater. Hydrobiologia, 463, 65-74.

Gluzman, C. (1991). Aquatic Oligochaeta from permanent ponds of Berisso, Buenos Aires, Argentina. Physis, (B) 47 (112), 7-9.

Gluzman, C. (1994). La drilofauna asociada a Eichhornia crassipes en ríos bonaerenses y su comportamiento alimentario. Physis, (B) 49 (116-117), 5-11.

Gorni, G. R., Alves, R. G. (2006). Naididae (Annelida, Oligochaeta) associated with Pomacea bridgesii (Reeve) (Gastropoda, Ampullaridae). Revista Brasileira de Zoología, 23(4), 1059-1061.

Gorni, G. R., Alves, R. G. (2012). Oligoquetos (Annelida, Clitellata) em um córrego neotropical: uma abordagem do mesohabitat. Iheringia, Sér. Zool. [online] vol.102, 106-110.

Gophen, M., Yehuda, Y., Malinkov, A., Degani, G. (1998). Food composition of the fish community in Lake Agmon. Hydrobiologia, 380, 49-57.

Hellawell, J. M. (1986). Biological indicators of freshwater pollution and environmental management. Elsevier Applied Science, London.

Jamieson, B. G. M. (1970). A taxonomic revision of the Oligochaete genus Eukerria Michaelsen, 1935 (Ocnerodrilinae, Megascolecidae). Bulletin of the British Museum. Natural History. Zoology, 20 (5), 131-172.

Johnson, R. K., Wiederholm, T., Rosenberg, D.M. (1993). Freshwater biomonitoring using individual organisms, populations, and species assemblages of benthic macroinvertebrates. En Freshwater Biomonitoring and Benthic Macroinvertebrates (40-158). New York, Chapman and Hall.

Kaster, J. L. (1989). Observations of predator-prey on dispersal of an oligochaete prey, Limnodrilus hoffmeisteri. Hydrobiologia, 180, 191-193.

Kennedy, C. R. (1965). The distribution and habitat of Limnodrilus Claparède (Oligochaeta: Tubificidae). Oikos, 16, 26-38.

Lang, C. (1990). Quantitative relationships between oligochaete communities and phosphorus concentrations in lakes. Freshwater Biology, 24, 327-334.

Lamarck, J. P. A. (1816). Ordre Troisième: Vers Hispides. Histoire naturelle des animaux sans vertèbres, 3, 221-234.

Learner, M. A. (1979). The distribution and ecology of the Naididae (Oligochaeta) which inhabit the filter-beds of sewage-works in Britain. Water Research, 13, 1291-1299.

Loden, M. S. (1974). Predation by chironomid (Diptera) larvae on oligochaetes. Limnology and Oceanography, 19, 156-159.

Maciorowski, A., Benfield, E., Hendricks, A. (1977). Species composition, distribution, and abundance of oligochaetes in the Kanawha River, West Virginia. Hydrobiologia, 54 (1), 81-91.

Marchese, M. R. (1987).The ecology of some Benthic Oligochaeta from the Parana River, Argentina. Hydrobiologia, 155, 209-214.

Marchese, M. R. (2009). Annelida Oligochaeta. En Macroinvertebrados bentónicos Sudamericanos: Sistemática y biología (551-565). Fundación Miguel Lillo, Tucumán, Argentina. 
Marcus, E. (1943). Sobre Naididae do Brasil. Boletin Facultad Filosofía Ciencias y Letras Universidade Sao. Paulo, 32 (7), 3-247.

Marcus, E. (1944). Sobre Oligochaeta límnicos do Brasil. Boletin Facultad Filosofía Ciencias y Letras Universidade Sao. Paulo, 43 (8), 5-135.

Martin, P, Martinez-Ansemil, E, Pinder, A, Timm, T, Wetzel, M.J. (2008). Global diversity of oligochaetous clitellates («Oligochaeta»; Clitellata) in freshwater. Hydrobiologia, 595, 117-127.

Martin, P, Martinez-Ansemil, E, Sambugar, B. (2010). The Baikalian genus Rhyacodriloides in Europe: phylogenetic assessment of Rhyacodriloidinae subfam. $n$. within the Naididae (Annelida). Zoologica Scripta, 39, 462-482.

Martin, P., Martinez-Ansemil, E., Pinder, A., Timm, T., Wetzel, M. J. (2016). World checklist of freshwater Oligochaeta species. World Wide Web electronic publication. Available online at http://fada.biodiversity.be/group/show/12 [date accessed]

Michaelsen, W. (1935). Earthworms from South Western Australia. Journal and Proceedings of the Royal Society of Western Australia, 21, 39-43.

Milbrink, G. (1994). Oligochaetes and water pollution in two deep Norwegian lakes. Hydrobiologia, 278, 213-222.

Müller, O. F. (1773). Vermium terrestrium et fluviatilium, seu Animalium Infusoriorum, Helminthicorum et Testaceorum, non marinorum, succincta Historia. Heineck and Faber. Havniae et Lipsiae, xxv +214 .

Odabaşi, S., Arslan, N., Cirik, S. (2017). A New Rhyacodrilin (Oligochaeta) Record (Bothrioneurum vejdovskyanum Štolc. 1886) for Turkey. Süleyman Demirel Üniversitesi Eðirdir Su Ürünleri Fakültesi Dergisi, 13 (2), 179-185.

Oken, L. von. (1815). Oligochaeta. Lehrbuch der Naturgeschichte, Zoologie, 3 (1), 313-363.

Rahman, M. M., Verdegem, M. C. J., Nagelkerke, L. A. J., Wahab, M. A., Milstein, A., Verreth, J. A. J. (2006). Growth, production and food preference of rohu Labeo rohita (H.) in monoculture and in polyculture with common carp Cyprinus carpio (L.) under fed and non-fed ponds. Aquaculture, 257, 359-372.

Ratzel, F. (1868). Beiträge zur anatomische und systematische Kenntnis der Oligochaeten Zeitschrift für wissenschaftliche Zoologie, 18, 563-591.

Riera, P., Juget, J., Martinet, F. (1991). Predator-prey interactions: effects of carp predation on Tubificid dynamics and carp production in experimental fishpond. Hydrobiologia, 226, 129-136.

Rodrigues, L. F. T., Santana, L. D. y Alves, R. G. (2013), Aquatic oligochaetes associated with bryophytes in an Atlantic Forest stream. Biota Neotropica, 13, 371-375.

Rodríguez Capítulo, A., Ocon, C., Tangorra, M., Paggi, A. C. Cortelezzi, R., Spaccesi, F. (2003). Estudios zoobentonicos recientes en el Río de la Plata. Biología Acuática, 21, 19-30.

Rodríguez, P., Fend, S. V. (2018). On spermatophore-producing aquatic microdrile oligochaetes. Zootaxa, 4497 (1), 041-060.

Rodríguez, P., Reynoldson, T. B. (2011). The Pollution Biology of Aquatic Oligochaetes, Springer Science+Business Media B.V. 
Rosenberg, M., Resh, V. H. (1993). Freshwater biomonitoring and benthic macroinvertebrates, Chapman and Hall, New York.

Schenková, J., Helešic, J., Jarkovský, J. (2006). Seasonal dynamics of Bythonomus lemani and Bothrioneurum vejdovskyanum (Oligochaeta, Annelida) in relation to environmental variables. Institute of Zoology, Slovak Academy of Sciences, Biologia, Bratislava, 61/5, 517-523.

Schürch, V. M., Walter, J. E. (1978). Diets of leeches (Hirudinea) and triclads (Turbellaria, Tricladida). Archiv fur Hydrobiology, 83 (2), 272-284.

Schwank, P. (1982). Turbellarien, Oligochaeten und Archianneliden des Breitenbach und anderer oberhessischer Mittelgebirgsbache. III. Die Taxozonosen der Turbellarien und Oligochaeten in Fliessgewassern - eine synokologische Gliederung. Schlitzer produktionsbiologische Studien (43-3). Archiv fur Hydrobiology, Suppl 62, 191-253.

Singer, R. (1978). Suction-feeding in Aeolosoma (Annelida). Transactions of the American Microscopical Society, 97, 105-111.

Stolc, A. (1886). Prehled ceských Tubificidu. Sitzungsberichte der Königlichen Böhemischen Gesellschaft der Wissenschaften. En Prag, Mathematisch-Naturwissenschaftliche Classe (640-647). Jahrgang.

Stephenson, J. (1932). Oligochaeta I. Microdrili, mainly Enchytraeidae. Discovery Reports, 4, 233-264.

Streit, B. (1977). Morphometric relationships and feeding habits of two species of Chaetogaster, Ch. limnaei and Ch. diastrophus (Oligochaeta). Archiv fur Hydrobiology, Suppl. 48, 424-437.

Teisaire, E. S., Aragón, M. López, García Moreno, A. (2005). The Gavrilov earthworms collection (Annelida, Oligochaeta). En Advances in Earthworms Taxonomy II. Proceeding of the $2^{\text {nd }}$ International Oligochaeta Taxonomy Meeting $\left(2^{\text {nd }}\right.$ IOTM). Cluj-University Press, Cluj-Napoca, Romania.

Timm, T. (2009). A guide to the freshwater Oligochaeta and Polychaeta of Northern and Central Europe. Lauterbornia, 66, 1-235.

Traunspurger, W., Drews, C. (1996). Toxicity analysis of freshwater and marine sediments with meio and macrobenthic organisms: a review. Hydrobiologia, 328, 215-261.

Trivinho-Strixino, S., Correia, L. C. S., Sonoda, K. (2000). Phytophilous Chironomidae (Diptera) and other macroinvertebrates in the ox-bow Infernão lake (Jataí Ecological Station, Luiz Antônio, SP, Brazil). Revista Brasileira de Biologia, São Carlos, 60 (3), 527-535.

Ude, H. (1896). Enchytraeiden. Die Oligochaeten des Südlichen Süd-Amerikas und geographische Beziehung der selben. Ergebnisse der Hamburger Malgalhaensischen Sammelreise, 1. Fridrichsen, Hamburg.

Uzunov, J., Košel, V., Sládeèek, V. (1988). Indicator value of freshwater Oligochaeta. Acta Hydrochimica et Hydrobiologica, 16 (2), 173-186.

Vejdovský, F. (1879). Beiträge zur vergleicheidenden Morphologhie der Annelinden: Monographie der Enchytraeiden. Verlag von F. Tempsky, Prag. 
Vejdovský, F. (1883). Revisio Oligochaetorum [Oligochaetarum] Bohemiae Sitzungsberichte der Königlichen Böhemischen Gesellschaft der Wissenschaften En Prag, Mathematisch-Naturwissenschaftliche Classe (213-228).

Verdonschot, P. F. M. (1999). Micro-distribution of oligochaetes in a softbottomed lowland stream (Elsbeek; Netherlands). Hydrobiologia, 406, 149-163.

Woodward, G., Papantoniou, G., Edwards, F., Lauridsen, R. B. (2008). Trophic trickles and cascades in a complex food web: impacts of a keystone predator on stream community structure and ecosystem processes. Oikos, 117 (5), 683692.

Yan, Y., Liang, Y. (2004). Energy flow in Branchiura sowerbyi (Oligochaeta: Tubificidae) in a shallow macrophyte-dominated lake, Biandantang Lake. Chinese. Journal of Oceanography and Limnolology, 22, 403-407.

Zilli, F. L., Montalto, L., Marchese, M. R. (2008). Benthic invertebrate assemblages and functional feeding groups in the Parana' River floodplain (Argentina). Limnologica, 38, 159-171.

Zeybek M, Kopal Pahin, S, Yýldýz, S. (2018). The Aquatic Oligochaeta (Annelida) Fauna of the Karasu Stream. LimnoFish, 4 (1), 30-35.doi: 10.17216/LimnoFish.363933. 